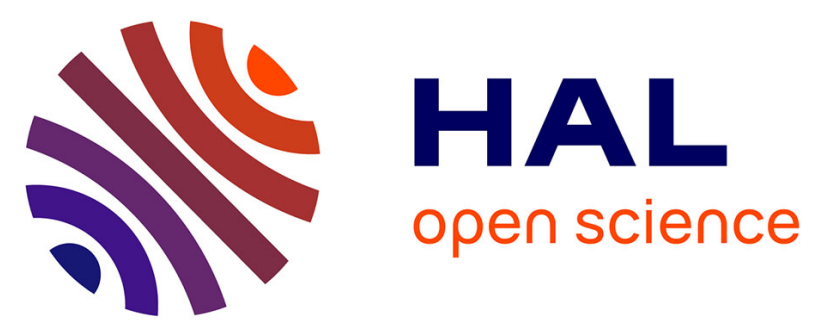

\title{
Examination of large-scale structures in a turbulent plane mixing layer. Part 2. Dynamical systems model
}

\author{
L. Ukeiley, L. Cordier, Remi Manceau, J. Delville, M. Glauser, J. Bonnet
}

\section{To cite this version:}

L. Ukeiley, L. Cordier, Remi Manceau, J. Delville, M. Glauser, et al.. Examination of large-scale structures in a turbulent plane mixing layer. Part 2. Dynamical systems model. Journal of Fluid Mechanics, 2001, 441, pp.67-108. 10.1017/S0022112001004803 . hal-02990873

\section{HAL Id: hal-02990873 https://hal.science/hal-02990873}

Submitted on 5 Nov 2020

HAL is a multi-disciplinary open access archive for the deposit and dissemination of scientific research documents, whether they are published or not. The documents may come from teaching and research institutions in France or abroad, or from public or private research centers.
L'archive ouverte pluridisciplinaire HAL, est destinée au dépôt et à la diffusion de documents scientifiques de niveau recherche, publiés ou non, émanant des établissements d'enseignement et de recherche français ou étrangers, des laboratoires publics ou privés.

\section{(ㅇ)(1) $\$$}

Distributed under a Creative Commons Attribution - NonCommercial - NoDerivatives $\mid 4.0$ 


\title{
Examination of Large-Scale Structures in a Turbulent Plane Mixing Layer. Part 2.
}

\section{Dynamical Systems Model}

\author{
By L. Ukeiley ${ }^{1} \dagger$, L. Cordier ${ }^{2}$, \\ J. Delville ${ }^{2}$ M. Glauser AND J. P. Bonnet ${ }^{2}$ \\ ${ }^{1}$ Clarkson University, Potsdam, NY \\ ${ }^{2}$ LEA/CEAT UMR CNRS 6609, Poitiers, France
}

(Received 14 December 1999)

The temporal dynamics of large-scale structures in a plane turbulent mixing layer are studied through the development of a low-order dynamical system of ordinary differential equations (ODEs). This model was derived by projecting the Navier-Stokes equations onto an empirical basis set from the Proper Orthogonal Decomposition (POD) using a Galerkin method. To obtain this low-dimensional set of equations, a truncation is performed including only the first POD mode for selected streamwise/spanwise $\left(k_{1} / k_{3}\right)$ modes. The initial truncations included only $k_{3}=0$. Once these truncations were evaluated, non-zero spanwise wavenumbers were then added. These truncated systems of equations are then examined in the pseudo Fourier space in which they are solved and by reconstructing the velocity field. Two different methods for closing the mean streamwise velocity are evaluated to show the importance for the dynamical representation of the flow to introduce in the low-order dynamical system a term allowing for feedback between

$\dagger$ Present address: National Research Council, NASA Langley Research Center, Mail Stop 166, Hampton, VA 23681 
the turbulent and mean flows. The results of the numerical simulations showed a strongly periodic flow indicative of the spanwise aligned vorticity. The simulated flow had the correct energy distributions in the cross-stream direction. These models also indicated that the events associated with the centre of the mixing layer lead the temporal dynamics. For truncations involving both spanwise and streamwise wavenumbers the reconstructed velocity field exhibited the main spanwise and streamwise vortical structures known to exist in this flow. The streamwise aligned vorticity was shown to connect spanwise vortex tubes.

\section{Introduction}

Understanding and modelling turbulent flows are becoming more important as many advanced applications require finer details of the turbulence to be known. At the same time it is becoming increasingly evident that large-scale organized motions present in turbulent flows influence many physical properties such as: mixing, noise, vibrations, heat transfer, drag, lift, etc. Therefore, understanding the dynamics of large-scale flow organizations will play a crucial role in the ability to understand turbulent flows. In that vein, the method offered in this study leads to a simple dynamical model, in comparison to the full Navier-Stokes equations, which can be used as a tool for understanding the dynamics of the flow and serve as a test bed for control applications.

The idea of applying a low-order dynamical system approach of the type discussed in this communication stems from the need to simplify the Navier-Stokes equations to a minimal set of ordinary differential equations (ODEs), able to describe correctly 
the essential dynamical behaviour of the flow. The approach is based on a small set of equations modelling some attractor (obtained through a truncation procedure based on the physics of a given flow) which has enough of the same characteristics of the NavierStokes equations and capture the underlying physical processes. The lower dimension of the new system would allow easier comprehension of the mechanisms in turbulence; thus utilizing the eigenfunctions of the Proper Orthogonal Decomposition (POD) for the basis set is beneficial. The POD, first introduced in fluid mechanics by Lumley (1967), provides an optimal basis set, in terms of kinetic energy representation, which can lead to the smaller system of equations.

Lorenz (1963) was the first to utilize a low-order dynamical system to study fluid mechanics. Based on physical approximations, he developed a set of three first-order differential equations to model the temperature and velocity field dynamics in a RayleighBénard convecting layer. Some years later, Ruelle \& Takens (1971) made the theoretical link between low-order dynamical systems and turbulence. They proposed that, in a certain bounded domain and under specific conditions, a mathematical object called a strange attractor which corresponds to turbulence might exist for the Navier-Stokes equations. This work led to many studies using dynamical systems techniques especially for closed flow systems. The following is some of the work using POD-Galerkin models for open flow systems. For a more comprehensive list see Berkooz, Holmes \& Lumley (1993a) or Delville (1995). A low-order dynamical system based on POD modes was first applied to an open turbulent system by Aubry et al. (1988). They developed a low-dimensional set of ODEs that model the near wall region of a turbulent boundary layer by using the experimentally determined eigenfunctions of Herzog (1986). Their model equations (hereafter called the Cornell model) exhibited several dynamical regimes (periodic, quasi- 
periodic, intermittent, chaotic) as the Heisenberg control parameter was varied. When the solutions of their model ODEs are used to reconstruct the three dimensional velocity fields in the wall region, they found results consistent with experimental observations in a turbulent boundary layer, i.e. the burst-sweep cycle. For a full review of their findings in the context of dynamical systems, the reader is referred to the recent book of Holmes, Lumley \& Berkooz (1996).

Based on the initial work of Aubry et al. (1988), a significant number of other studies has been undertaken. Aubry, Lumley \& Holmes (1990) modeled drag reduction on the wall region by applying stretching transformations to the eigenfunctions of the Cornell model. This study suggests that the intermittent events observed in the original model can not be considered as an artifact of the closure assumption but are deeply rooted in the dynamical phenomenon of the wall region. Berkooz, Holmes \& Lumley (1991) generalized the wall layer Cornell model to permit uncoupled evolution of streamwise and cross-stream disturbances. Their main conclusion was that the intermittent behaviour reported in Aubry et al. (1988) is a direct consequence of the ODE's invariant subspaces and symmetries which reflect natural physical symmetries of the flow. Sanghi \& Aubry (1993) investigated the persistency of the intermittent behaviour observed in the model of Aubry et al. (1988) when streamwise variations which were not accounted for in an explicit way in the Cornell model are now considered. With this higher order model, the intermittent behaviour persisted, but with higher additional complexities.

Since the original work of the Cornell group, others have examined the role of coherent structures in the wall region of a turbulent boundary layer with a similar approach. To avoid introducing an inhomogeneous pressure term at the boundary as was done in the Cornell model, Zhou \& Sirovich (1992) constructed 'wall' eigenfunctions with full 
channel validity. In order to facilitate comparisons with the results of the Cornell model, they first adopt the same severe truncation: five modes with no streamwise variation. This five mode model equations displayed intermittent, quasiperiodic, and chaotic behaviour similar to that of the Cornell group. When propagating modes (streamwise dependent modes) are included in the model, a physically more realistic dynamical behaviour is then obtained. They concluded that the propagating modes lie more at the heart of the triggering mechanism for the bursting process than does the idea of pressure fluctuations proposed previously by Aubry et al. (1988). To get a better understanding of the final stages of transition in a boundary layer, Rempfer (1995) derived dynamical models for different regions of the flow, by Galerkin projection of the Navier-Stokes equations onto the POD eigenfunctions extracted from numerically computed flow fields.

Free shear flows have been studied using the POD-Galerkin type models in both jets and mixing layers. A low-order dynamical system model was developed for the axisymmetric jet by Glauser, Zheng \& Doering (1989) and Zheng \& Glauser (1991). The eigenfunctions utilized in these studies have been extracted from two-point velocity measurements in the mixing layer of a high Reynolds number axisymmetric jet (see Glauser \& George 1992 and Glauser 1987). Glauser, Zheng \& George (1990) postulated a spatially evolving dynamical system model for the axisymmetric jet mixing layer. In this model, the mean velocity quantities would be solved simultaneously, thus resulting in equations of a different form from that of the temporally evolving case. Rajaee, Karlsson \& Sirovich (1994) applied the snapshot form of POD to measurements obtained in a non-turbulent forced mixing layer. Since their flow was forced through phase aligning the measurements, they were able to compute the time dependence of the POD random coefficients directly by projection of the snapshots on the eigenfunctions and to compare them with the result 
of the low-dimensional model. They found good agreement between the model and the direct projection, serving as justification for the low-dimensional description.

This study is the continuation of the work presented in Delville et al. (1998) that discussed the experimental measurements and the results from applying the POD to the plane turbulent mixing layer. Using the POD modes found in that study, a lowdimensional dynamical system model is developed in this communication by utilizing a Galerkin projection of the POD eigenfunctions on the Navier-Stokes equations. Due to the orthogonality condition of the eigenfunctions, this yields an ODE for each streamwise/spanwise wavenumber pair kept in a given truncation. Several versions of the model are presented for severely truncated systems. The temporal dynamics of the mixing layer are studied through examination of the POD coefficients and by transforming the results to real space and examining the velocity field.

\section{Application of the POD to the plane turbulent mixing layer}

Discussion of the application of the POD to the mixing layer is detailed in the first part of this paper (Delville et al. 1998); however, it is briefly reiterated here for continuity of this work.

Lumley (1967) suggested that the coherent structure should be the deterministic structure $\psi_{i}(\vec{x}, t)$ which has the largest mean square projection on the random velocity field $u_{i}(\vec{x}, t)$. Maximizing the mean square projection via the calculus of variations leads to the following integral eigenvalue problem:

$$
\int_{\mathcal{T}} \int_{\mathcal{D}} R_{i j}\left(\vec{x}, \vec{x}^{\prime}, t, t^{\prime}\right) \psi_{j}\left(\vec{x}^{\prime}, t^{\prime}\right) \mathrm{d} \vec{x}^{\prime} \mathrm{d} t^{\prime}=\lambda \psi_{i}(\vec{x}, t) .
$$


The symmetric kernel of this Fredholm integral equation is the velocity cross-correlation tensor $R_{i j}$ defined by:

$$
R_{i j}\left(\vec{x}, \vec{x}^{\prime}, t, t^{\prime}\right)=\left\langle u_{i}(\vec{x}, t) u_{j}\left(\vec{x}^{\prime}, t^{\prime}\right)\right\rangle
$$

where the angle brackets denote the appropriate average for the problem under consideration (see $§ 3.1$ for a discussion on how averaging was performed in the mixing layer).

If the random field is statistically homogeneous or periodic in one or more directions or stationnary in time, the eigenfunctions $\psi_{i}(\vec{x}, t)$ become Fourier modes (Lumley 1970 , George 1988), so that the POD reduces to the harmonic decomposition in these directions. In the mixing layer under study, the spanwise direction is considered to be homogeneous allowing Fourier analysis in this direction. As detailed in Delville et al. (1998), time is mapped to the streamwise direction through Taylor's Hypothesis thus forcing this direction to be considered stationary and allowing for Fourier analysis of the streamwise direction. The eigenvalue problem (2.1) can then now be written as

$$
\int_{\mathcal{D}} \boldsymbol{\Psi}_{i j}\left(x_{2}, x_{2}^{\prime} ; k_{1}, k_{3}\right) \Phi_{j}^{(n)}\left(x_{2}^{\prime} ; k_{1}, k_{3}\right) \mathrm{d} x_{2}^{\prime}=\lambda^{(n)}\left(k_{1}, k_{3}\right) \Phi_{i}^{(n)}\left(x_{2} ; k_{1}, k_{3}\right),
$$

where $\boldsymbol{\Psi}_{i j}\left(x_{2}, x_{2}^{\prime} ; k_{1}, k_{3}\right)$ is the cross-spectral tensor, defined as the streamwise and spanwise Fourier transform of the cross-correlation tensor. The above equation is the one solved to extract the eigenfunctions used in the models developed in the next section.

As pointed out in Lumley (1967), the properties of the integral equation (2.3) are governed by the Hilbert-Schmidt theory. One of the most interesting property is that the eigenfunctions form a complete orthonormal set, which means that the Fourier transform in the $x_{1}$ and $x_{3}$ directions of the fluctuating random field can be reconstructed in the following way:

$$
\widetilde{u}_{i}\left(k_{1}, k_{3} ; x_{2}, t\right)=\left(L_{1} L_{3}\right)^{\frac{1}{2}} \sum_{n=1}^{N_{P O D}} a_{k_{1}, k_{3}}^{(n)}(t) \Phi_{i}^{(n)}\left(x_{2} ; k_{1}, k_{3}\right),
$$


where $a_{k_{1}, k_{3}}^{(n)}(t)$ are time random coefficients. This relationship can be transformed back into physical space by the two dimensional Fourier transform defined by:

$$
u_{i}\left(x_{1}, x_{2}, x_{3}, t\right)=\int_{-\infty}^{+\infty} \int_{-\infty}^{+\infty} \widetilde{u}_{i}\left(k_{1}, k_{3} ; x_{2}, t\right) \mathrm{e}^{+2 \pi j\left(k_{1} x_{1}+k_{3} x_{3}\right)} \mathrm{d} k_{1} \mathrm{~d} k_{3} .
$$

In (2.4), $L_{1}$ and $L_{3}$ are the spatial extent of the domain $\mathcal{D}$ in the $x_{1}$ and $x_{3}$ directions, respectively, and $N_{P O D}$ is the number of POD modes.

The coefficient $a_{k_{1}, k_{3}}^{(n)}(t)$ can be determined from (2.4) by:

$$
a_{k_{1}, k_{3}}^{(n)}(t)=\frac{1}{\left(L_{1} L_{3}\right)^{\frac{1}{2}}} \int_{\mathcal{D}} \widetilde{u}_{i}\left(k_{1}, k_{3} ; x_{2}, t\right) \Phi_{i}^{(n) *}\left(x_{2} ; k_{1}, k_{3}\right) \mathrm{d} x_{2} .
$$

The low-order dynamical systems that will be developed in the following section solve for the temporal evolution of the coefficients $a_{k_{1}, k_{3}}^{(n)}$ in $(2.4)$. Therefore, in the context of a deterministic dynamical model, the temporal coefficients now become deterministic functions of time. The time traces of these coefficients serve as 'building blocks' to study the temporal evolution of the large-scale structures. One of the needs for developing a dynamical system model of the expansion coefficients is that the integral in (2.6) cannot be solved unless the whole decomposition domain is known simultaneously which was not the case for this study. Experimentally, obtaining the full field measurements is extremely difficult although the recent work of Citriniti (1996) has shown that it is possible. Those experiments demonstrated there is a wealth of information that can be gained to complement the dynamical systems model approaches. 


\section{Temporal dynamical equations}

This section details the derivation of the equations for the dynamical model. The method used here is similar to that of Aubry et al. (1988) and Glauser et al. (1989) which has recently been documented by Holmes, Lumley \& Berkooz (1996). In the following section, two sets of equations are derived with their differences stemming from the closure method for the mean streamwise velocity. In the both methods ( $\$ 3.2 .1$ ), the mean velocity is calculated from a Boussinesq approximation. The first method yields a mean velocity which is calculated a priori and is held constant throughout the time integrations. In the other method ( $\$ 3.2 .2)$, a cutoff wavenumber will be chosen and the contribution to the mean velocity from wavenumbers lower than this wavenumber are assumed to be steady, while the contribution from wavenumbers greater than the cutoff will have their effects vary with time. The difference between these two methods will become apparent in the following discussion. The need to have some contribution to a time dependent mean has been discussed in Holmes, Lumley \& Berkooz (1996) and will become apparent in $\S 6$ where the linear stability of the trivial solution is discussed.

\subsection{Momentum equations}

In the same manner as detailed in Berkooz, Holmes \& Lumley (1993b) and Aubry et al. (1988), two different types of averaging operators will be used. In the following sections: the time average denoted by an overbar and a spatial average in the $x_{1}$ and $x_{3}$ directions denoted by $\langle\bullet\rangle$ :

$$
\begin{gathered}
\overline{(\bullet)}=\frac{1}{T} \int(\bullet) \mathrm{d} t, \\
\langle\bullet\rangle=\frac{1}{L_{1} L_{3}} \int(\bullet) \mathrm{d} x_{1} \mathrm{~d} x_{3} .
\end{gathered}
$$


An overbarred quantity depends on $x_{1}, x_{2}, x_{3}$ but not on $t$, while a $\langle\bullet\rangle$ quantity depends on $x_{2}$ and $t$ but not on $x_{1}$ and $x_{3}$.

The first step is to perform a Reynolds decomposition of the Navier-Stokes equations $\left(u_{i}=\left\langle u_{i}\right\rangle+u_{i}^{\prime}\right.$ where $\left.\left\langle u_{i}\right\rangle=U_{i}\right)$. It should be noted that we are applying to the Ergodic Hypothesis to swap between the spatial averages in the derived equations and the block averages which were used to acquire the experimental data. For an incompressible fluid, without body forces, the following equations are obtained:

$$
\frac{\partial u_{i}^{\prime}}{\partial t}+u_{j}^{\prime} \frac{\partial u_{i}^{\prime}}{\partial x_{j}}-\left\langle u_{j}^{\prime} \frac{\partial u_{i}^{\prime}}{\partial x_{j}}\right\rangle+u_{2}^{\prime} \frac{\partial U_{1}}{\partial x_{2}} \delta_{i 1}+U_{1} \frac{\partial u_{i}^{\prime}}{\partial x_{1}}=-\frac{1}{\rho} \frac{\partial p^{\prime}}{\partial x_{i}}+\nu \frac{\partial^{2} u_{i}^{\prime}}{\partial x_{j} \partial x_{j}}
$$

The following two assumptions have been made in reducing (3.3) from the NavierStokes equations:

(a) $U_{3} \approx 0$

(b) $U_{2} \approx 0$.

These two assumptions corresponds to a thin layer assumption which is fairly standard for the mixing layer. The first one is explicitly forced by the symmetries which were applied to the experimental data. The second assumption has been examined, and it was shown that the terms involving $U_{2}$ were small in comparison to the other terms kept in the model. Finally, the mean velocity is such that $U_{1}=U_{1}\left(x_{2}, t\right)$.

\subsection{Eddy viscosity representation}

In this section a relationship will be developed for the mean streamwise velocity present in (3.3). This relation is necessary because, in the severely truncated system which will be modeled the measured mean velocity profile will be incorrect. Moreover, since there 
is no direct way to represent the mean velocity in terms of the POD modes, this study will use an eddy viscosity relationship to balance the mean streamwise velocity with the Reynolds stress a term which can easily be written in terms of POD modes. Two different ways of representing Reynolds stresses in terms of the POD modes will be discussed thus introducing the two different mean velocity closures.

Assuming that the Reynolds stresses act like the viscous stresses, Boussinesq (1877) developed a relationship where the Reynolds stresses are directly proportional to the velocity gradient. This relationship can be written for the mean streamwise velocity as follows:

$$
U_{1}\left(x_{2}\right)=-\frac{1}{\nu_{e}} \int_{0}^{x_{2}} \overline{u_{1}^{\prime} u_{2}^{\prime}} \mathrm{d} x_{2}^{\prime}+U_{1}(0)
$$

where the Reynolds stresses can be written in terms of the POD modes as,

$$
\overline{u_{1}^{\prime} u_{2}^{\prime}}=\sum_{i=1}^{N_{P O D}} \iint_{-\infty}^{\infty} \lambda_{k_{1}, k_{3}}^{(i)} \Phi_{1, k_{1}, k_{3}}^{(i)} \Phi_{2, k_{1}, k_{3}}^{(i) *} \mathrm{~d} k_{1} \mathrm{~d} k_{3}
$$

In (3.4) $\nu_{e}$ is determined from the Free Shear layer model as formulated by PrandtlReichardt: $\nu_{e}=K \delta_{w}\left(U_{a}-U_{b}\right)$ where $U_{a}-U_{b}$ represents the velocity difference, $\delta_{w}$ represents the vorticity thickness and the constant $K$ is 0.01 for the mixing layer. This approximation forces a constant eddy viscosity across the layer. Although $\nu_{e}$ is not truly constant across the mixing layer the value from this relationship was found to be approximately equal to the integral value of $\nu_{e}$ calculated from (3.4) with experimental data (see Ukeiley 1995). In (3.4), $U_{1}(0)$ is set to be equal to $U_{m}=\frac{U_{a}+U_{b}}{2}$ to preserve the convection velocity of the experimental system in our model. We will see in $\$ 6.2 .1$ that this term is important for the spectral behaviour of our low-order system.

Before the actual relationships used in this study are derived, it is important to 
point out why it is beneficial to have some feedback between the turbulent and mean velocity. It is well known that the mean gradient contributes to the turbulence through the production process. However, many researchers in turbulence also feel that turbulent velocities must affect the mean velocities in some way. In Wick, Glauser \& Ukeiley (1994) the effect of the presence of a coherent structure on the mean streamwise velocity profile was studied through the use of Pseudo Flow Visualization in a turbulent axisymmetric jet mixing layer. It is clearly evident from that work that the presence or absence of a structure alters the mean velocity profile. Following in this vein, the present study seeks to incorporate feedback between the turbulence and the mean. The need for feedback from the turbulence to the mean was also discussed in Holmes, Lumley \& Berkooz (1996). They stressed that in the dynamical model of Aubry et al. (1988) the system grew unbounded without feedback which is similar to the results for this model discussed in $\S 7$.

\subsubsection{No-feedback relationship}

In this set of equations, a steady mean velocity is calculated and its numerical value used in (3.3). This value is calculated from only the modes kept in the truncation as follows:

$U_{1}\left(x_{2}\right)=-\frac{1}{\nu_{e}} \int_{0}^{x_{2}}\left[\sum_{i=1}^{N_{P O D}} \int_{-k_{1 T}}^{k_{1 T}} \int_{-k_{3 T}}^{k_{3 T}} \lambda_{k_{1}, k_{3}}^{(i)} \Phi_{1, k_{1}, k_{3}}^{(i)} \Phi_{2, k_{1}, k_{3}}^{(i) *} \mathrm{~d} k_{1} \mathrm{~d} k_{3}\right] \mathrm{d} x_{2}^{\prime}+U_{1}(0)$,

where $k_{1 T}$ and $k_{3 T}$ represent the largest streamwise and spanwise wavenumbers kept in the truncation and $N_{P O D}$ will be set to one. Even though there is no mechanism for feedback between the turbulent and mean velocity, the amplitude of the mean velocity should be appropriately scaled for the severely truncated systems studied here. This is very important because the system of equations is driven by the production term and in 
order for it not to overwhelm the system, i.e. unbounded growth, this term must be of the correct order.

\subsubsection{Filter relationship}

In this set of equations, (3.4) is split into a steady and time dependent part. This will be done by choosing cutoff wavenumbers in both $k_{1}$ and $k_{3}$. These cutoff wavenumbers $\left(k_{1 C}\right.$ and $\left.k_{3 C}\right)$ act as a filter point, and the contribution to the mean streamwise velocity from wavenumbers less than the cutoff values will be considered steady while the contribution from wavenumbers greater than the cutoff values will be used for feedback between the turbulent and mean velocities. The set $E^{C \leqslant}$, the ensemble of the modes $\left(k_{1}, k_{3}\right)$ less than the cutoff values is defined by $E^{C \leqslant}=\left\{k_{1}, k_{3} ;\left|k_{1}\right| \leqslant k_{1 C}\right.$ and $\left.\left|k_{3}\right| \leqslant k_{3 C}\right\}$ and the set $E^{C>}$ of the wavenumbers kept in the truncation but with wavenumbers greater than the cutoff values $E^{C>}=\left\{k_{1}, k_{3} ; k_{1 T} \geqslant\left|k_{1}\right|>k_{1 C}\right.$ and $\left.k_{3 T} \geqslant\left|k_{3}\right|>k_{3 C}\right\}$. This technique of spectral decomposition is applied to the mean velocity profile to yield:

$$
U_{1}\left(x_{2}, t\right)=U_{1 F}\left(x_{2}\right)+U_{1 \text { uns }}\left(x_{2}, t\right) .
$$

$U_{1 F}\left(x_{2}\right)$ is estimated using (3.6) with the modes $\left(k_{1}, k_{3}\right) \in E^{C \leqslant}$ and $U_{1}$ uns $\left(x_{2}, t\right)$ can be written as:

$$
\begin{gathered}
U_{1 \text { uns }}\left(x_{2}, t\right)=-\frac{1}{\left(L_{1} L_{3}\right) \nu_{e}} \times \\
\int_{0}^{x_{2}}\left[\sum_{i=1}^{N_{P O D}} \iint_{\left(k_{1}, k_{3}\right) \in E^{C>}} a_{k_{1}, k_{3}}^{(i)}(t) a_{k_{1}, k_{3}}^{(i) *}(t) \Phi_{1, k_{1}, k_{3}}^{(i)}\left(x_{2}\right) \Phi_{2, k_{1}, k_{3}}^{(i) *}\left(x_{2}\right) \mathrm{d} k_{1} \mathrm{~d} k_{3}\right] \mathrm{d} x_{2}^{\prime},
\end{gathered}
$$

by taking into account $(2.4)$. 
Substituting (3.7) into (3.3) yields

$$
\begin{gathered}
\frac{\partial u_{i}^{\prime}}{\partial t}+u_{j}^{\prime} \frac{\partial u_{i}^{\prime}}{\partial x_{j}}-\left\langle u_{j}^{\prime} \frac{\partial u_{i}^{\prime}}{\partial x_{j}}\right\rangle+u_{2}^{\prime} \frac{\partial U_{1} u n s}{\partial x_{2}} \delta_{i 1}+U_{1} \text { uns } \frac{\partial u_{i}^{\prime}}{\partial x_{1}}+u_{2}^{\prime} \frac{\partial U_{1 F}}{\partial x_{2}} \delta_{i 1}+U_{1 F} \frac{\partial u_{i}^{\prime}}{\partial x_{1}} \\
=-\frac{1}{\rho} \frac{\partial p^{\prime}}{\partial x_{i}}+\nu \frac{\partial^{2} u_{i}^{\prime}}{\partial x_{j} \partial x_{j}} .
\end{gathered}
$$

In this equation there is a fixed portion of the mean streamwise velocity which forces a constant (non-time dependent) production term combined with a relationship that allows for feedback between the turbulent and mean velocities at higher wavenumbers. In the limit that $k_{1 C}$ and $k_{3 C}$ are set to zero one arrives at a set of equations where all of the represented mean velocity is allowed to vary with time. This limiting case is examined as the feedback relationship in $\$ 6$ and in Ukeiley (1995).

\subsection{Fourier transform and Galerkin projection}

Before the Galerkin projection is performed two steps must be taken. The first step is to take the two dimensional Fourier transform of (3.9). The second is to expand the Fourier coefficients of the of the velocity in terms of POD modes using (2.4).

After the two previous operations were performed to the manipulated Navier-Stokes equations, a Galerkin projection was applied. As defined in Aubry et al. (1988), this projection is represented by the following inner product:

$$
\left(N, \Phi^{(l)}\right)=\int_{\mathcal{D}} N_{i, k_{1}, k_{3}}\left(t, x_{2}\right) \Phi_{i, k_{1}, k_{3}}^{(l) *}\left(x_{2}\right) \mathrm{d} x_{2}=0 \quad l=1, \cdots, N_{g a l}
$$

where $N_{i, k_{1}, k_{3}}\left(t, x_{2}\right)$ represents the Fourier transform of the Navier-Stokes equations and $N_{\text {gal }}$ the number of Galerkin modes kept in the dynamical system. Before the final equations were achieved, one other relationship was applied to the equations; this was 
the orthogonality relationship of the eigenfunctions:

$$
\int_{\mathcal{D}} \Phi_{i, k_{1}, k_{3}}^{(n)} \Phi_{i, k_{1}, k_{3}}^{(l) *} \mathrm{~d} x_{2}=\delta_{n l}
$$

After some analytical manipulations, (3.9) can be rewritten in a general form as:

$$
\begin{aligned}
\frac{\mathrm{d} a_{k_{1}, k_{3}}^{(n)}}{\mathrm{d} t}(t)= & \sum_{m=1}^{N_{g a l}} \Lambda_{k_{1}, k_{3}}^{(m)} a_{k_{1}, k_{3}}^{(m)}(t)+\sum_{p, q=1}^{N_{g a l}} \sum_{k_{1}^{\prime}, k_{3}^{\prime}} Q_{k_{1}^{\prime}, k_{3}^{\prime}, k_{1}, k_{3}}^{(p)(q)} a_{k_{1}^{\prime}, k_{3}^{\prime}}^{(p)}(t) a_{k_{1}-k_{1}^{\prime}, k_{3}-k_{3}^{\prime}}^{(q)}(t) \\
& +\sum_{p, q, r=1}^{N_{g a l}} \sum_{k_{1}^{\prime}, k_{3}^{\prime}} C_{k_{1}^{\prime}, k_{3}^{\prime}, k_{1}, k_{3}}^{(p)(q)(r)} a_{k_{1}^{\prime}, k_{3}^{\prime}}^{(p)}(t) a_{k_{1}^{\prime}, k_{3}^{\prime}}^{(q) *}(t) a_{k_{1}, k_{3}}^{(r)}(t) \\
& -\frac{1}{\rho} \int_{\mathcal{D}} \frac{\partial \tilde{p}_{k_{1}, k_{3}}}{\partial x_{i}} \Phi_{i, k_{1}, k_{3}}^{(n) *}\left(x_{2}\right) \mathrm{d} x_{2} .
\end{aligned}
$$

Since the eigenfunctions are divergence free and the streamwise and spanwise directions are assumed to be homogeneous, the pressure term in (3.12) can be written as:

$$
\int_{\partial \mathcal{D}} \tilde{p}_{k_{1}, k_{3}}\left(x_{2}\right) \boldsymbol{\Phi}_{k_{1}, k_{3}}^{(n) *} \cdot \boldsymbol{n} \mathrm{d} S=\left[\widetilde{p}_{k_{1}, k_{3}}\left(x_{2}\right) \Phi_{2, k_{1}, k_{3}}^{(n) *}\left(x_{2}\right)\right]_{-L_{2}}^{L_{2}}
$$

where $\partial \mathcal{D}$ is the boundary domain and $\boldsymbol{n}$ the outer normal on $\partial \mathcal{D}$. If decay of the eigenfunctions is assumed at infinity, we can write that $\Phi_{2, k_{1}, k_{3}}^{(n)}\left(x_{2}\right)=0$ at $x_{2}=-L_{2}$ and $x_{2}=L_{2}$. The result is that the pressure term can be eliminated in the rest of the analysis contrary to the case of Aubry et al. (1988) where the effect of the pressure term on the outer layer needed to be modeled.

In the following two subsections, the dynamical equations will be shown for the two different mean velocity closures discussed in $§ 3.2 .1$ and 3.2 .2 . 


\subsection{No-feedback relationship}

For the no-feedback relationship, (3.12) can be written in a simplified form as follows:

$$
\begin{aligned}
\frac{\mathrm{d} a_{k_{1}, k_{3}}^{(n)}}{\mathrm{d} t}(t)= & \sum_{m=1}^{N_{g a l}}\left(\Lambda_{k_{1}, k_{3}}^{1(m)}+\Lambda_{k_{1}, k_{3}}^{2(m)}\right) a_{k_{1}, k_{3}}^{(m)}(t) \\
& +\sum_{p, q=1}^{N_{g a l}} \sum_{k_{1}^{\prime}, k_{3}^{\prime}} Q_{k_{1}^{\prime}, k_{3}^{\prime}, k_{1}, k_{3}}^{(p)(q)} a_{k_{1}^{\prime}, k_{3}^{\prime}}^{(p)}(t) a_{k_{1}-k_{1}^{\prime}, k_{3}-k_{3}^{\prime}}^{(q)}(t),
\end{aligned}
$$

where the coefficients $\Lambda_{k_{1}, k_{3}}^{1(m)}, \Lambda_{k_{1}, k_{3}}^{2(m)}$ and $Q_{k_{1}^{\prime}, k_{3}^{\prime}, k_{1}, k_{3}}^{(p)(q)}$ are calculated from the POD eigenfunctions. The exact form of these coefficients is given in appendix A.

The first two terms on the right hand side of (3.14) are linear. The first term $\Lambda^{1}$ is a direct result of the viscous diffusion term in (3.9) (term $\left.\nu \frac{\partial^{2} u_{i}^{\prime}}{\partial x_{j} \partial x_{j}}\right)$. The second term $\Lambda^{2}$ results from the production and convection terms (one part comes from the term $u_{2}^{\prime} \frac{\partial U_{1}}{\partial x_{2}} \delta_{i 1}$ and the other part comes from the term $U_{1} \frac{\partial u_{i}^{\prime}}{\partial x_{1}}$ of (3.9) and contains the mean velocity calculated from (3.6). The next term $Q$ is a quadratic term. This term is representative of the fluctuation interactions and exhibits the transfer of energy between the Fourier and POD modes in the dynamical system (terms $u_{j}^{\prime} \frac{\partial u_{i}^{\prime}}{\partial x_{j}}-\left\langle u_{j}^{\prime} \frac{\partial u_{i}^{\prime}}{\partial x_{j}}\right\rangle$ of (3.9). 


\subsection{Filter relationship}

For this relationship, the equation can be written as follows:

$$
\begin{aligned}
\frac{\mathrm{d} a_{k_{1}, k_{3}}^{(n)}}{\mathrm{d} t}(t)= & \sum_{m=1}^{N_{g a l}}\left(\Lambda_{k_{1}, k_{3}}^{1(m)}+\Lambda_{k_{1}, k_{3}}^{2(m)}\right) a_{k_{1}, k_{3}}^{(m)}(t) \\
& +\sum_{p, q=1}^{N_{g a l}} \sum_{k_{1}^{\prime}, k_{3}^{\prime}} Q_{k_{1}^{\prime}, k_{3}^{\prime}, k_{1}, k_{3}}^{(p)(q)} a_{k_{1}^{\prime}, k_{3}^{\prime}}^{(p)}(t) a_{k_{1}-k_{1}^{\prime}, k_{3}-k_{3}^{\prime}}^{(q)}(t) \\
& +\sum_{p, q, r=1}^{N_{g a l}} \sum_{\left(k_{1}^{\prime}, k_{3}^{\prime}\right) \in E^{C>}} C_{k_{1}^{\prime}, k_{3}^{\prime}, k_{1}, k_{3}}^{((p)(q)(r)} a_{k_{1}^{\prime}, k_{3}^{\prime}}^{(p)}(t) a_{k_{1}^{\prime}, k_{3}^{\prime}}^{(q) *}(t) a_{k_{1}, k_{3}}^{(r)}(t) .
\end{aligned}
$$

For this closure assumption, the linear term $\Lambda_{k_{1}, k_{3}}^{1(m)}$ is equal to the one in the nofeedback case $(\S \mathrm{A} .1)$. The other linear term $\Lambda_{k_{1}, k_{3}}^{2(m)}$ is similar to the one in (3.14) except now $\Lambda^{2}$ uses the filtered mean streamwise velocity $U_{1 F}$ which is calculated using (3.6) with the modes $\left(k_{1}, k_{3}\right) \in E^{C \leqslant}$. The quadratic term $Q_{k_{1}^{\prime}, k_{3}^{\prime}, k_{1}, k_{3}}^{(p)(q)}$ is the same as in (3.14). The last term $C_{k_{1}^{\prime}, k_{3}^{\prime}, k_{1}, k_{3}}^{(p)(q)(r)}$ is cubic and is a result of the modeling of the mean velocity. One part comes from the term $u_{2}^{\prime} \frac{\partial U_{1 u n s}}{\partial x_{2}} \delta_{i 1}$ and the other part comes from the term $U_{1 u n s} \frac{\partial u_{i}^{\prime}}{\partial x_{1}}$ of (3.9) where $U_{1 \text { uns }}$ is calculated from (3.8). This term represents the interaction between the mean velocity and the turbulent fluctuations with the summations performed only over values greater than the filter setting and not the whole truncated domain. The cubic coefficients are shown in appendix A.2.

\section{Turbulent viscosity model}

In order to limit the degrees of freedom in the dynamical system, the number of POD and streamwise/spanwise wavenumber modes kept in the model will be truncated. 
Due to the rapid convergence of the POD modes (49\% of the turbulent kinetic energy is contained in the first mode, Delville et al. 1998), we will use only the first one. Hence, from here, the superscript denoting POD mode will be dropped and the first POD mode can be assumed.

A consequence of the truncation of streamwise/spanwise wavenumbers is that the dynamical system is no longer complete because it will not account for the energy transfer between the modes kept and those lost to the truncation. As a first attempt to account for this, a Heisenberg spectral model (Hinze 1975) similar to the one utilized by Aubry et al. (1988) and Glauser et al. (1989) will be used. In this approach, it is assumed that the small scales (neglected modes) remove energy from the larger ones (the modes kept) by a global viscous action of kinematic turbulence viscosity $\nu_{T}$. See appendix B for details of the method to estimate $\nu_{T}$.

In introducing the turbulent viscosity $\nu_{T}$ into the dynamical equations, (3.12) can now be written as:

$$
\begin{aligned}
\frac{\mathrm{d} a_{k_{1}, k_{3}}}{\mathrm{~d} t}(t)= & {\left[\left(1+\alpha \frac{\nu_{T}}{\nu}\right) \Lambda_{k_{1}, k_{3}}^{1}+\Lambda_{k_{1}, k_{3}}^{2}\right] a_{k_{1}, k_{3}}(t) } \\
& +\sum_{k_{1}^{\prime}, k_{3}^{\prime}} Q_{k_{1}^{\prime}, k_{3}^{\prime}, k_{1}, k_{3}} a_{k_{1}^{\prime}, k_{3}^{\prime}}(t) a_{k_{1}-k_{1}^{\prime}, k_{3}-k_{3}^{\prime}}(t) \\
& +\sum_{k_{1}^{\prime}, k_{3}^{\prime}} C_{k_{1}^{\prime}, k_{3}^{\prime}, k_{1}, k_{3}}\left|a_{k_{1}^{\prime}, k_{3}^{\prime}}(t)\right|^{2} a_{k_{1}, k_{3}}(t),
\end{aligned}
$$

where $C_{k_{1}^{\prime}, k_{3}^{\prime}, k_{1}, k_{3}}$ is zero for the no-feedback case. The coefficient $\alpha$ controls the portion of energy which the small scales, neglected by the truncation, acquire. In terms of dynamical systems, $\alpha$ is considered like a bifurcation parameter. Large values of $\alpha$ correspond to a stable system. 


\section{General properties of the ODEs and solutions}

This section details some of the properties of the differential equations along with the properties of the numerical integrations.

\subsection{Symmetry properties}

To solve the equations derived in the previous section it will be necessary to map some quantities to negative wavenumbers using symmetry properties of the flow field. For the eigenfunctions the applied symmetries are based on the relationships for the crossspectral tensor as shown in Cordier (1996). The following symmetries are used for the eigenfunctions:

$$
\begin{aligned}
& \Phi_{1,-k_{1},-k_{3}}=\Phi_{1, k_{1}, k_{3}}^{*} ; \Phi_{2,-k_{1},-k_{3}}=\Phi_{2, k_{1}, k_{3}}^{*} ; \Phi_{3,-k_{1},-k_{3}}=\Phi_{3, k_{1}, k_{3}}^{*}, \\
& \Phi_{1,-k_{1}, k_{3}}=\Phi_{1, k_{1}, k_{3}}^{*} ; \Phi_{2,-k_{1}, k_{3}}=\Phi_{2, k_{1}, k_{3}}^{*} \quad ; \quad \Phi_{3,-k_{1}, k_{3}}=-\Phi_{3, k_{1}, k_{3}}^{*}, \\
& \Phi_{1, k_{1},-k_{3}}=\Phi_{1, k_{1}, k_{3}} \quad ; \quad \Phi_{2, k_{1},-k_{3}}=\Phi_{2, k_{1}, k_{3}} \quad ; \quad \Phi_{3, k_{1},-k_{3}}=-\Phi_{3, k_{1}, k_{3}} .
\end{aligned}
$$

The expansion coefficients $a_{k_{1}, k_{3}}(t)$ are instantaneous quantities, and there is no explicit reason that they should be forced to satisfy the above stated symmetries at all times. However, due to the fact that the velocity is real, certain constraints can be imposed on these coefficients. Utilizing the symmetries on the eigenfunctions it can be shown that:

$$
a_{k_{1}, k_{3}}(t)=a_{-k_{1},-k_{3}}^{*}(t)
$$

\subsection{Solution domain}

For truncations involving several $k_{1}$ values with $k_{3}=0$, only positive values of $k_{1}$ are solved; and the others are extracted from the application of the above symmetry on the 


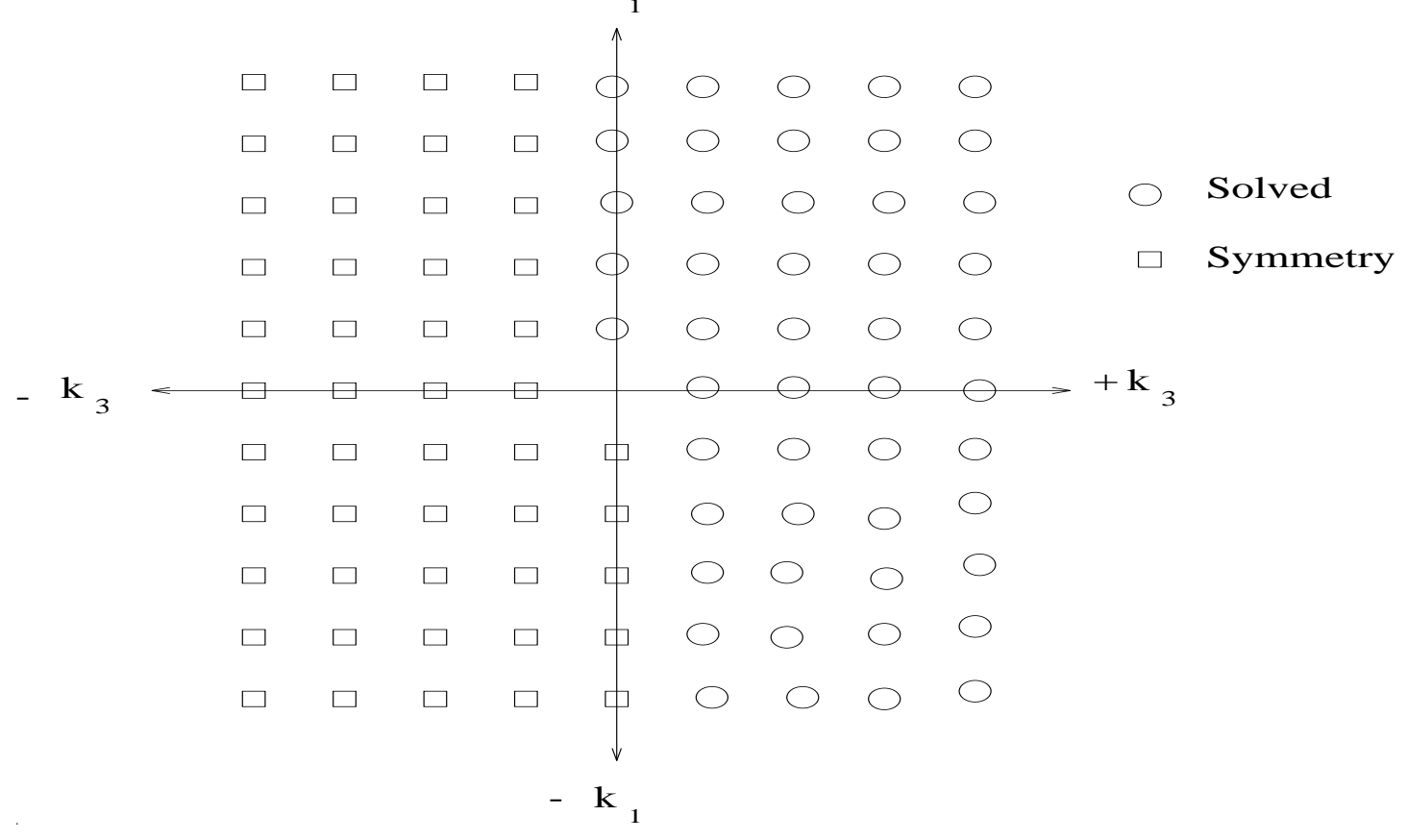

FIGURE 1. Solution domain

$a$ 's. When truncations involved wavenumbers of $k_{3} \neq 0$ the solution expands dramatically. In these cases $\pm k_{1}$ is solved along with $+k_{3}$. The solution domain is graphically depicted in figure 1.

Due to the fact that the only symmetry involved relates the diagonal quadrants, the choice of solution domain is rather arbitrary. The solution domain could have easily been the inverse: $\pm k_{3}$ is solved along with $+k_{1}$. Checking this, in fact, showed the domain solution chosen did not affect the solutions.

\subsection{The zero wavenumber coefficient}

The zero wavenumber mode $\left(k_{1}=0, k_{3}=0\right)$ needs to be considered independently in the truncation. Due to (5.2), the coefficient $a_{0,0}$ is real. The temporal evolution is given 
by equation:

$$
\frac{\mathrm{d} a_{0,0}}{\mathrm{~d} t}=\left(1+\alpha \frac{\nu_{T}}{\nu}\right) \Lambda_{0,0}^{1} a_{0,0}
$$

where the coefficient $\Lambda_{0,0}^{1}$ is found numerically to be strictly negative.

Starting from (4.1), the previous equation can be obtained after the introduction of different properties (see appendix A for the expression of the dynamical system coefficients):

(a) The factor $\left(1-\delta_{k_{1}, 0} \delta_{k_{3}, 0}\right)$ in front of the quadratic term implies that the contribution of this term is zero for the $\left(k_{1}=0, k_{3}=0\right)$ mode.

(b) The cubic term, is either equal to zero (for the no-feedback case) or is composed of two terms. In front of the first term, there is a factor $\left(2 \pi j k_{1}\right)$, making this term equal to zero for $k_{1}=0$. In the second term the eigenfunction $\Phi_{2, k_{1}, k_{3}}$ appears causing it to be zero.

The continuity equation in the spectral domain can be written as:

$$
\left(2 \pi \jmath k_{1}\right) \Phi_{1, k_{1}, k_{3}}\left(x_{2}\right)+\frac{\partial}{\partial x_{2}} \Phi_{2, k_{1}, k_{3}}\left(x_{2}\right)+\left(2 \pi \jmath k_{3}\right) \Phi_{3, k_{1}, k_{3}}\left(x_{2}\right)=0 .
$$

For $k_{1}=k_{3}=0$, this equation becomes:

$$
\frac{\partial}{\partial x_{2}} \Phi_{2, k_{1}, k_{3}}\left(x_{2}\right)=0
$$

and the eigenfunction $\Phi_{2, k_{1}, k_{3}}\left(x_{2}\right)$ is constant. We remind the reader that in $\S 3.3$, we assumed that $\lim _{x_{2} \rightarrow \pm \infty} \Phi_{2, k_{1}, k_{3}}\left(x_{2}\right)=0$; the consequence is that $\Phi_{2, k_{1}=0, k_{3}=0}\left(x_{2}\right)=0$. Finally, the contribution of the cubic term in the feedback case is also equal to zero.

Equation (5.3) expressed the fact that the amplitude of the mode $a_{0,0}(t)$ decreased 
exponentially to zero with a time constant given by:

$$
\tau_{0,0}=-\frac{1}{\left(1+\alpha \frac{\nu_{T}}{\nu}\right) \Lambda_{0,0}^{1}}
$$

Therefore, this mode can only influence the dynamical evolution of the system for a short transient $\uparrow$. From now on, the amplitude of the coefficient $a_{0,0}(t)$ will be assumed to be zero, and the mode $\left(k_{1}=0, k_{3}=0\right)$ will not be retained in the truncation.

\subsection{Initial conditions}

The initial conditions for the system of equations is based on the magnitude of the

eigenvalues. Essentially since $a$ goes like $(\lambda)^{\frac{1}{2}}$ the initial values for the coefficients will be determined by the following equationț:

$$
\operatorname{Re}\left(a_{k_{1}, k_{3}}^{(1)}(t=0)\right)=\operatorname{Im}\left(a_{k_{1}, k_{3}}^{(1)}(t=0)\right)=\left(\frac{\lambda_{k_{1}, k_{3}}^{(1)}}{2}\right)^{\frac{1}{2}} .
$$

Using this value for the initial conditions the simulations should start with approximately the correct magnitude. In the discussion of the simulations this will help to serve as a criteria to evaluate the results.

The effects of varying the initial conditions have been studied and shown to have little effect on the results. A change in amplitude of the initial conditions, by as much as an order of magnitude, only affected the amount of time before the results settled into the same behaviour.

$\dagger$ However, we have to keep in mind that for the very low values of the control parameter $\alpha$, the time constant $\tau_{0,0}$ may be very high.

$\ddagger$ In this equation, Re and Im denote the real and imaginary part of a complex number respectively. 
5.5. Numerics

To investigate the temporal evolution of the coefficients, a Runge-Kutta technique was utilized. The particular Runge-Kutta method used was a $5^{\text {th }} / 6^{\text {th }}$ order Verner method from the IMSL numerical libraries. This particular method was chosen for ease of application along with its ability to multi-step between prescribed time steps. Several other Runge-Kutta methods were tried and shown to give the same quantitative results.

A minimum time step of $8.10^{-5}$ seconds was chosen. This time step was used in order to minimize the intermittent stepping. Since for the truncations studied here one should not see frequencies greater than $1000 \mathrm{~Hz}$; therefore, the selected step-size allows for adequate resolution of any waves observed.

Since the Runge-Kutta method used can only handle real valued numbers, the coefficients are split into their real and imaginary parts,

$$
a_{k_{1}, k_{3}}(t)=a_{k_{1}, k_{3}}^{r}(t)+\jmath a_{k_{1}, k_{3}}^{i}(t) .
$$

The number of degrees of freedom for the system then becomes twice the modes included in the system. Computationally this involved twice the number of equations; however, in the function evaluation for the Runge-Kutta method the real and imaginary parts were combined and complex manipulations were used.

Computations were performed on an IBM RS6000 550 work station using double precision arithmetic. 


\section{Linear stability analysis of the trivial solution}

In order to understand the influence of the two different closure assumptions, the no feedback relationship ( $\$ 3.2 .1$ ) and filter relationship ( $\$ 3.2 .2$ ), on the dynamical behaviour of the low-order system and the role of the different terms involved in (4.1), a linear stability analysis of the trivial solution have been done. The turbulent viscosity model introduced in appendix $\mathrm{B}$ is function of the specific truncation used in the lowdimensional system. Then, to permit this study, the factor $\alpha \frac{\nu_{T}}{\nu}$ of (4.1) will be replaced in this section by $100 \beta$.

\subsection{Analysis method}

The time evolution equation of a particular mode $\left(k_{1}, k_{3}\right)$ assumed without interaction with other modes is given by:

$$
\frac{\mathrm{d} a_{k_{1}, k_{3}}}{\mathrm{~d} t}=\left[(1+100 \beta) \Lambda_{k_{1}, k_{3}}^{1}+\Lambda_{k_{1}, k_{3}}^{2}\right] a_{k_{1}, k_{3}}+C_{k_{1}, k_{3}}\left|a_{k_{1}, k_{3}}\right|^{2} a_{k_{1}, k_{3}}
$$

If the complex number $a_{k_{1}, k_{3}}$ is written in its polar form as $r_{k_{1}, k_{3}} \mathrm{e}^{3 \theta_{k_{1}, k_{3}}}$, and $\Lambda_{k_{1}, k_{3}}^{1, r}$, $\Lambda_{k_{1}, k_{3}}^{2, r}, C_{k_{1}, k_{3}}^{r}$ and $\Lambda_{k_{1}, k_{3}}^{1, i}, \Lambda_{k_{1}, k_{3}}^{2, i}, C_{k_{1}, k_{3}}^{i}$ are the real and imaginary part of the terms $\Lambda_{k_{1}, k_{3}}^{1}, \Lambda_{k_{1}, k_{3}}^{2}, C_{k_{1}, k_{3}}$ respectively.

Linearizing (6.1) around the trivial solution yields the following equations:

$$
\begin{aligned}
& \frac{\mathrm{d} r_{k_{1}, k_{3}}}{\mathrm{~d} t}=\left[(1+100 \beta) \Lambda_{k_{1}, k_{3}}^{1, r}+\Lambda_{k_{1}, k_{3}}^{2, r}\right] r_{k_{1}, k_{3}}, \\
& \frac{\mathrm{d} \theta_{k_{1}, k_{3}}}{\mathrm{~d} t}=\left[(1+100 \beta) \Lambda_{k_{1}, k_{3}}^{1, i}+\Lambda_{k_{1}, k_{3}}^{2, i}\right] .
\end{aligned}
$$

The linear stability of the trivial solution is then directly determined by the sign of 
the linear growth rate defined as:

$$
\sigma_{k_{1}, k_{3}}=\operatorname{Re}\left(\Lambda_{k_{1}, k_{3}}\right)=\operatorname{Re}\left((1+100 \beta) \Lambda_{k_{1}, k_{3}}^{1}+\Lambda_{k_{1}, k_{3}}^{2}\right)
$$

where $\operatorname{Re}\left(\Lambda_{k_{1}, k_{3}}^{1}\right)<0$ for each mode $\left(k_{1}, k_{3}\right)$. If $\operatorname{Re}\left(\Lambda_{k_{1}, k_{3}}^{2}\right)$ have negative values then the linear growth rate $\sigma_{k_{1}, k_{3}}$ remains negative for all values of the control parameter $\beta$ (the term $(1+100 \beta)$ is assumed to be strictly positive). At the opposite, if $\operatorname{Re}\left(\Lambda_{k_{1}, k_{3}}^{2}\right)$ is positive, there exist a critical value $\beta_{k_{1}, k_{3}}^{c}$ such that the linear growth rate becomes equal to zero:

$$
\beta_{k_{1}, k_{3}}^{c}=-\frac{1}{100}\left(\frac{\operatorname{Re}\left(\Lambda_{k_{1}, k_{3}}^{2}\right)}{\operatorname{Re}\left(\Lambda_{k_{1}, k_{3}}^{1}\right)}+1\right)
$$

The response of the mode $\left(k_{1}, k_{3}\right)$ to a small disturbance can then be determined in the following manner:

- If $\sigma_{k_{1}, k_{3}}<0$ or $\beta>\beta_{k_{1}, k_{3}}^{c}$, every harmonic disturbance is exponentially damped and the mode $\left(k_{1}, k_{3}\right)$ is said stable.

- If $\sigma_{k_{1}, k_{3}}>0$ or $\beta<\beta_{k_{1}, k_{3}}^{c}$, every harmonic disturbance is exponentially amplified and the mode $\left(k_{1}, k_{3}\right)$ is said unstable.

- If $\sigma_{k_{1}, k_{3}}=0$ or $\beta=\beta_{k_{1}, k_{3}}^{c}$, the mode $\left(k_{1}, k_{3}\right)$ is said marginally stable.

\subsection{No feedback relationship}

Figure 2 shows $\dagger$ the surface of marginal stability when (4.1) is closed with a constant streamwise velocity. Several observations can be made:

$\dagger$ On this figure, $\delta k_{1} \exp$ and $\delta k_{3}$ exp correspond respectively to the experimental step in streamwise and spanwise directions (see on $\S 7.1$ for the definition and numerical values of these quantities). 


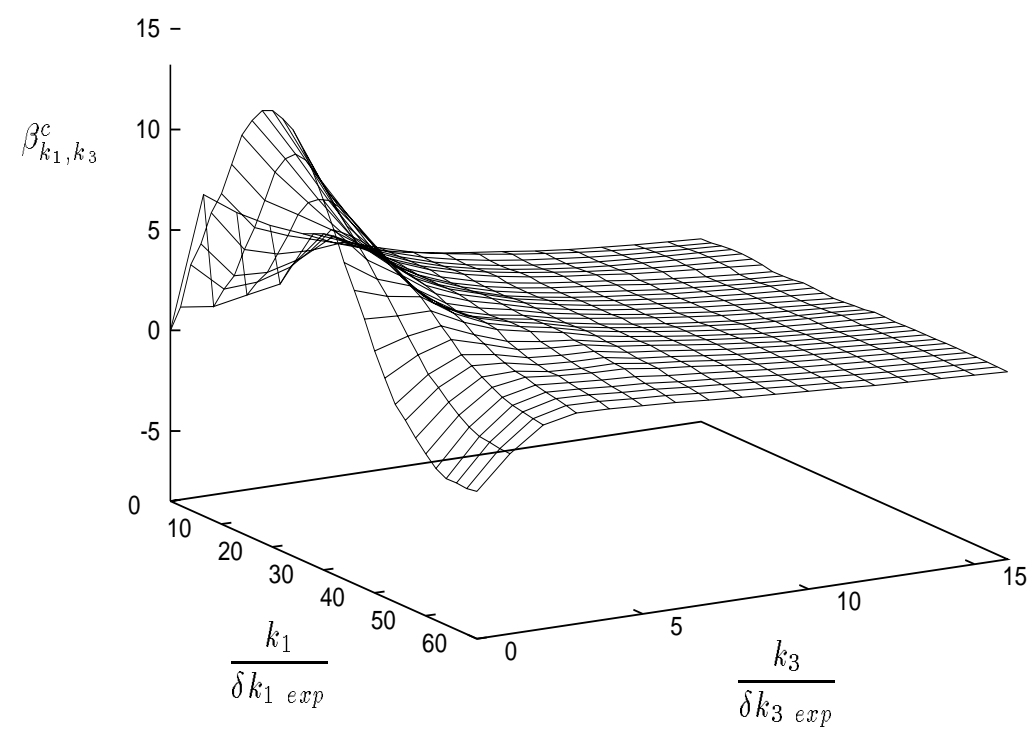

FIgURE 2. Marginal stability surface. No feedback relationship.

(a) When $\beta$ is sufficiently high, all the modes are stable, then the trivial solution is stable.

(b) When $\beta$ decreases, there is a mode $\left(k_{1}, k_{3}\right)$ which is the first to become unstable. This particular mode is called the most unstable mode.

(c) When $\beta$ is lower than this critical value, there is an ensemble of modes $\left(k_{1}, k_{3}\right)$ which are unstable at the same time.

(d) The most unstable modes are found for $k_{3}=0$ (2 dimensional modes). This result is compatible with the Squire theorem.

(e) When $\beta$ is even lower than this value and tends to zero, there is a great number of unstable modes $\left(k_{1}, k_{3}\right)$.

In the rest of this section, we will restrict ourselves to the case where the most unstable modes occur: $k_{3}=0$. In figure 3 , the linear growth rate variation, function of the non-dimensional streamwise wavenumber $\frac{k_{1}}{\delta k_{1} \text { exp }}$, is represented for different values of 


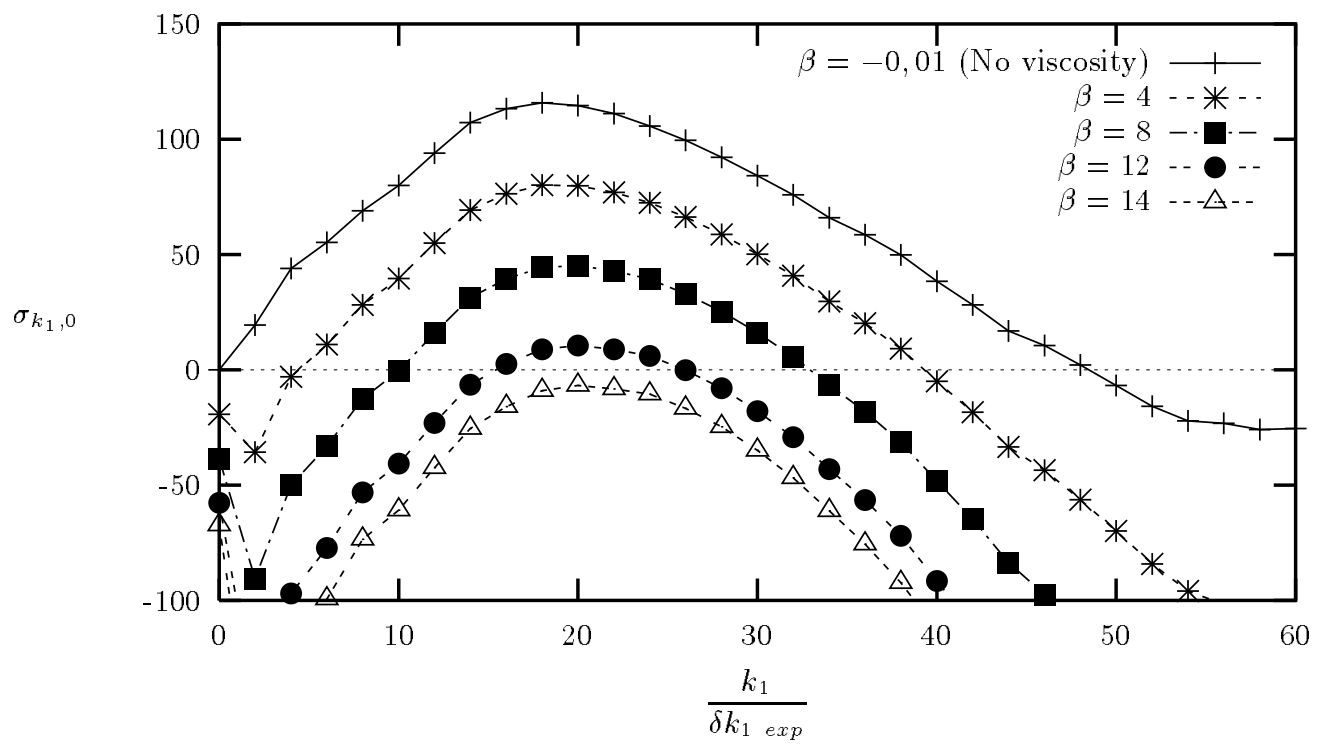

FigurE 3. Linear growth rate of the 2 dimensional modes $\left(k_{3}=0\right)$ for several values of the control parameter $\beta$. No feedback relationship.

the control parameter $\beta$. When $\beta=14$, the linear growth rate is negative for every value of $k_{1}$ : all the modes are stable, the trivial solution is then stable. When $\beta$ decreases the curve seems globally translated to the upper side. The streamwise wavenumber $\frac{k_{1}}{\delta k_{1} \text { exp }} \simeq 20$ is the first mode to cross the horizontal axis, then this is the most unstable mode. This particular mode becomes unstable for $\beta \simeq 13,2$.

Figure 4 compares the linear growth rate obtained for an inviscid mixing layer by Michalke (1964) to the one obtained with the simplified dynamical system. An excellent agreement is observed. Both the most linear unstable mode $k_{1}^{\max }$ and the value of its linear growth rate being comparable. It should be noted an important point: the dynamical system obtained from the POD is only based on the two point correlation tensor, (by opposition to Michalke's approach where the stability of the profile of the longitudinal velocity was in concern). This is a striking result that both approaches lead to similar results. 


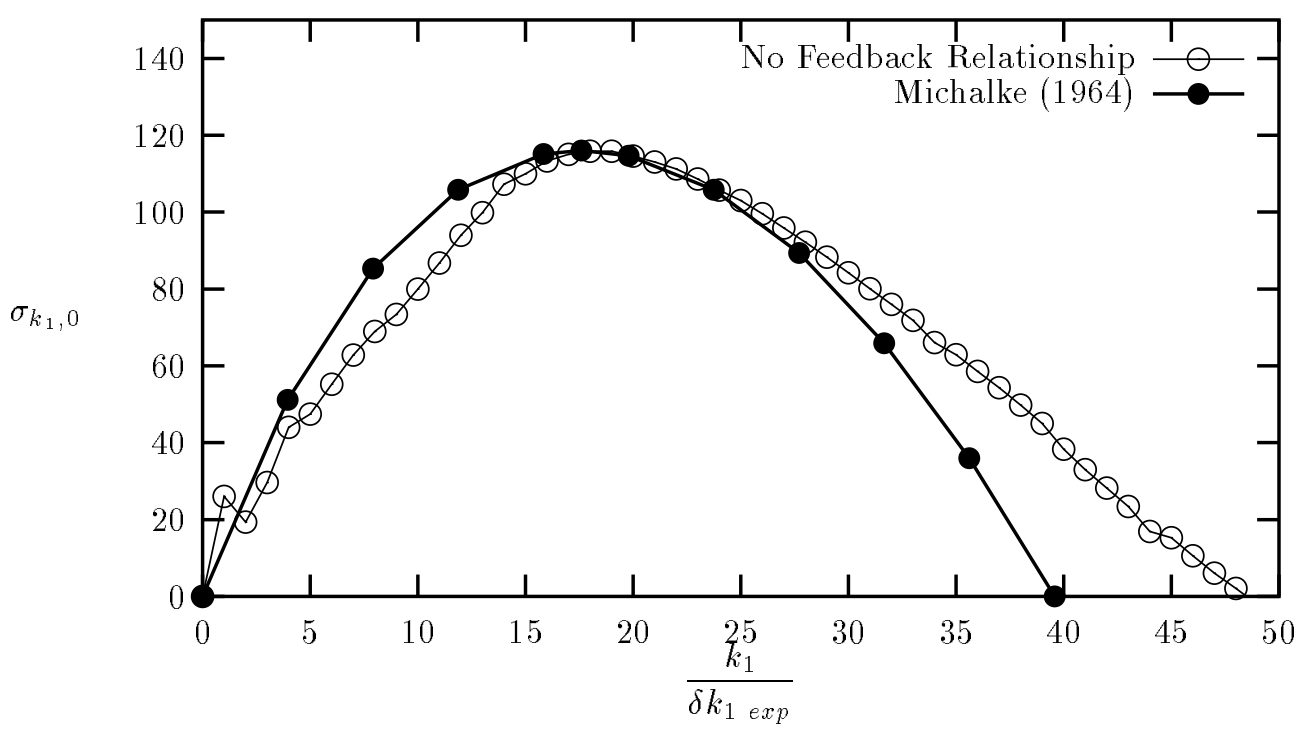

FigURE 4. Linear growth rate of the inviscid turbulent plane mixing layer. No feedback relationship.

This point confirms that the first POD mode is more than solely useful to describe the energy content of the flow but contains the necessary information for the linear stability description too. This shows further evidence why a low-order dynamical system approach based only on the first POD mode could be fruitful to follow the dynamical evolution of the flow under concern.

\subsubsection{Spectral behaviour}

From (6.2), we conclude that the natural frequency of a 2 dimensional mode $\left(k_{1}, 0\right)$ is given, in first approximation $\dagger$, by:

$$
f_{k_{1}, 0}=\frac{\operatorname{Im}\left(\Lambda_{k_{1}, 0}\right)}{2 \pi}=\frac{\operatorname{Im}\left[(1+100 \beta) \Lambda_{k_{1}, 0}^{1}+\Lambda_{k_{1}, 0}^{2}\right]}{2 \pi} .
$$

This equation points out that $f_{k_{1}, 0}$ is function of the control parameter $\beta$. How$\dagger$ The behaviour of the full dynamical system will not be exactly this one because, through the intermediary of the quadratic terms, the mode $\left(k_{1}, 0\right)$ is influenced by the other modes. 
ever, the numerical values of $\operatorname{Im}\left(\Lambda_{k_{1}, 0}^{1}\right)$ are lesser than $10^{-7}$ and the numerical values of $\operatorname{Im}\left(\Lambda_{k_{1}, 0}^{2}\right)$ are at least equal to $10^{2}$. Hence, for reasonable values of the control parameter, the natural frequency can be approximized with a good accuracy by: $f_{k_{1}, 0}=\frac{\operatorname{Im}\left(\Lambda_{k_{1}, 0}^{2}\right)}{2 \pi}$. Figure 5 represents the evolution of this frequency function of $k_{1,0}$.

Michalke (1964) demonstrated that, when the mean velocity profile is antisymmetric with respect to $y=0$, the instabilities phase velocity is independent of $k_{1}$ and is equal to $U_{1}(0)$. In a fixed reference frame, these instabilities, convected by the flow, are seen to move at frequency $U_{m} k_{1}$. On figure 5 , we observe that the natural frequency of the flow have comparable values with the theoretical ones. This result is a posterior justification of the use as mean velocity profile in (3.4) of $U_{1}(0)=U_{m}$.

Lastly, in figure 5 is represented the evolution of $U_{c} k_{1}$ where $U_{c}$ is the flow convection velocity determined in Delville et al. (1998). We find that the variation of $f_{k_{1}, 0}$ is better described by the term $U_{c} k_{1}$ than by the term $U_{m} k_{1}$. This last result is particularly true in the interval $[50,80]$ where $U_{c}$ exhibits a constant value equal to $0,8 U_{m}$.

\subsection{Feedback relationship. $k_{1 C}=k_{3 C}=0$}

If $k_{1 C}=k_{3 C}=0$, the steady part of the mean velocity profile is $U_{1 F}\left(x_{2}\right) \equiv U_{1}(0)$ and the linear term $\Lambda_{k_{1}, k_{3}}^{2}$ defined in appendix A.1 is then equal to:

$$
\Lambda_{k_{1}, k_{3}}^{2}=-\left(2 \pi j k_{1}\right) U_{m}
$$

This represents the limiting case of the filter relationship where the mean velocity is allowed to vary solely as a function of time.

This linear term is pure imaginary, recalling that $\operatorname{Re}\left(\Lambda_{k_{1}, k_{3}}^{1}\right)<0$ for each mode 


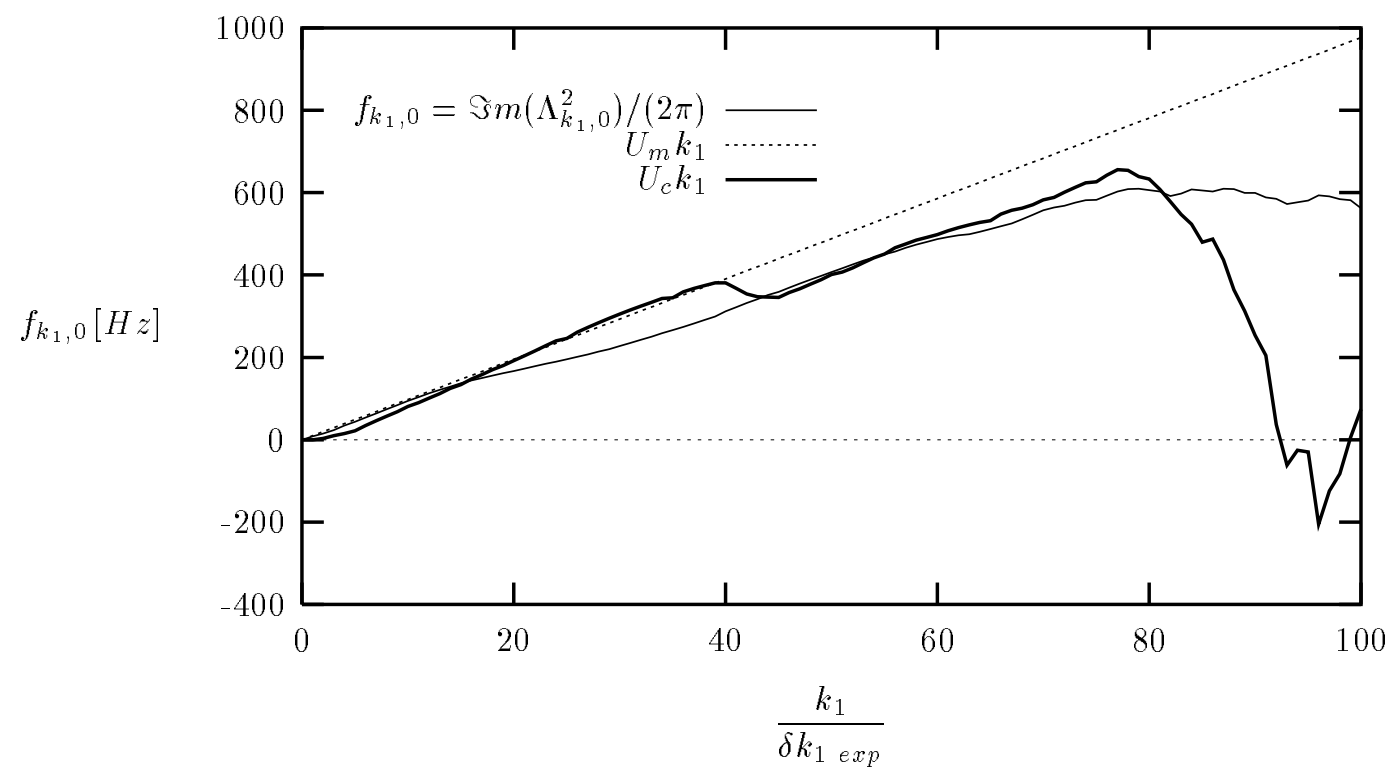

Figure 5. Natural frequencies of the low-order dynamical system. No feedback relationship.

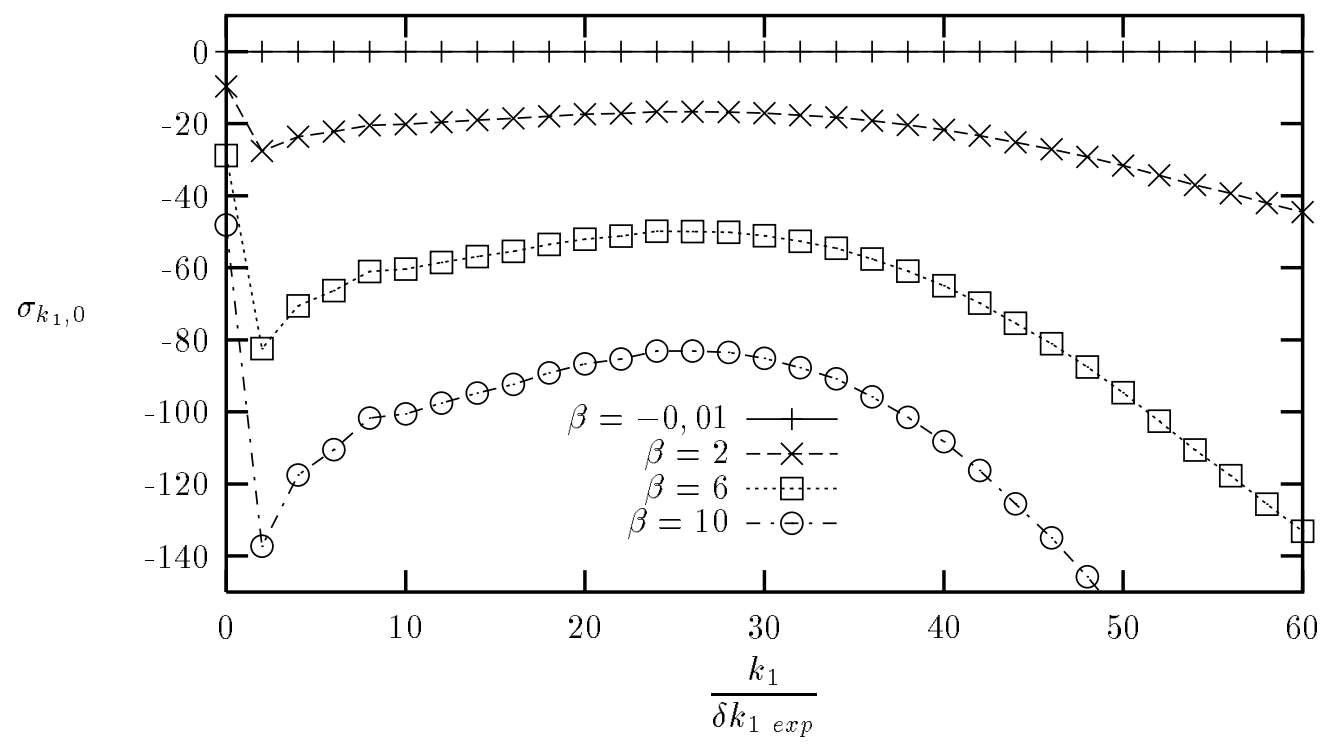

FiguRE 6 . Linear growth rate of the 2 dimensional modes $\left(k_{3}=0\right)$ for several values of the control parameter $\beta$. (For $\beta=-0,01$ no viscosity). Feedback relationship.

$\left(k_{1}, k_{3}\right)$ this implies that the trivial solution is stable whatever the control parameter $\beta$ is (see figure 6 for the most unstable modes, the two dimensional ones $k_{3}=0$ ). In this case, the marginal stability surface is defined by the horizontal plane of equation $\beta_{k_{1}, k_{3}}^{c}=-0,01$. 


\subsubsection{Global stability}

The question we want to address in this section is the following: does the attraction basin of the trivial solution cover all the space of initial conditions?

The previous results of linear stability analysis showed that, varying the control parameter $\beta$ from the trivial solution, no bifurcation is found. To confirm this result, an analysis of the system understudy was done (see Cordier 1996). Using the bifurcation package AUTO94 developed by Doedel, Keller \& Kernevez (1991), no other branch of solution was found. If the low-order dynamical system could exhibit an isolated branch then the previous argument would be wrong. However, we assumet that this is not the case for the system 4.1. The long term evolution of the system is then either the trivial solution, either unbounded growth to infinity. If all the cubic term of the loworder dynamical system would be negative, the solutions would converge to zero and the system would be called globally stable. Unfortunately, contrary to the original study of Aubry et al. (1988), this is not the case here. The global stability of the system is not assured and some initial conditions could lead to a divergence to infinity of the solutions. The conclusion is that the attraction basin of the trivial solution doesn't cover the space of all the initial conditions.

\subsubsection{Spectral behaviour}

In this section, we consider only two dimensional modes $k_{3}=0$. The natural frequency $f_{k_{1}, 0}$ of these modes is still defined by (6.5) where for the same reason as in $\S 6.2 .1$, the contribution of the linear term $\Lambda_{k_{1}, 0}^{1}$ can be neglected. Finally, the natural $\dagger$ All the numerical integrations that have been done confirm this assumption. 
frequency writes:

$$
f_{k_{1}, 0}=\frac{\operatorname{Im}\left(\Lambda_{k_{1}, 0}^{2}\right)}{2 \pi}=U_{m} k_{1}
$$

Since $\Lambda_{k_{1}, 0}^{2}$ has the same form similar results to those in section 6.2 .1 are found.

When $k_{1 C}=k_{3 C}=0$, the feedback relationship closure equation is not of interest in a physical point of view because the system can only, either converge to the trivial solution, either grows unbounded to infinity. When $k_{1 C} \neq 0$ or/and $k_{3 C} \neq 0$, the linear term $\Lambda_{k_{1}, k_{3}}^{2}$ is no more pure imaginary (see A 2$)$ and linear unstable modes $\left(k_{1}, k_{3}\right)$ appear for the trivial solution.

These results shows the justification for the filter technique if one wants to allow for feedback from the turbulence to the mean velocity.

\section{Solution for truncations with $k_{3}=0$}

Simulations of (4.1) using both the No-Feedback relationship and the filter technique, will be presented in this section. In addition to running the simulations for the two sets of equations, two different truncations with only $k_{3}=0$ modes were evaluated. The first truncation, detailed in this work, involved seven modes. In the second the system was shrunk to five modes while keeping the domain size $L_{1}$ the same. This second truncation was studied in Ukeiley (1995) and will not be presented here. The essential result is that this smaller system had essentially the same dynamics as the larger system but would allow for the inclusion of less modes when the inclusion of non-zero spanwise wavenumbers are included. For all the simulations involving only $k_{1}$, a spanwise domain size, $L_{3}$, of $0.27 \mathrm{~m}$ was used. This spanwise domain size was chosen because it is the physical domain 
of the experiments. The choice of domain size was shown to have no effect on the observed dynamics of the zero streamwise wavenumber simulations of Aubry et al. (1988).

\subsection{7 mode model no-feedback}

The wavenumbers included in the system are defined as an integer times the experimental step $\delta k_{1 \text { exp }}=\frac{\Delta f}{U_{c}}=0.29 \mathrm{~m}^{-1}$ and $\delta k_{3 \exp }=\Delta k_{z}=3.70 \mathrm{~m}^{-1}$ where $\Delta f$ and $\Delta k_{z}$ are, respectively, the minimal step size in frequency $f$ and spanwise wavenumber $k_{z}$ dictated by the experimental arrangement. From here on, only the integer value will be used and the appropriate $\delta$ will be assumed.

In this truncation with no spanwise wavenumbers $k_{3}=0$, the streamwise spacing is $10 \times \delta k_{1 \text { exp }}$. The included streamwise wavenumbers are the modes $k_{1}=10,20,30,40$, 50, 60, 70. The streamwise domain size $L_{1}$, is then $0.345 \mathrm{~m}$. Table 4 in appendix $\mathrm{C}$ shows the initial conditions used for the simulation. With a vorticity thickness of $28 \mathrm{~mm}$ and considering an average structure has an aspect ratio of 4 or 5 , the numerical window studied should contain at least two structures.

It is important to recall that the mean streamwise velocity used in (4.1) is not the measured quantity, but rather it is calculated from (3.6). For the particular truncation studied here the integration in $k_{1}$ runs from 10 to 70 . Figure 7 shows the velocity used for these simulations. In this figure and the ones to follow, $x_{2} / \delta_{\omega}$ will be denoted by $y+$. When comparing the profile in figure 7 to the measured values (see Delville et al. 1998), it is obvious that the convection velocity is preserved; however, the magnitudes on the low and high speed sides are significantly larger and smaller, respectively. This scales the production term, since it is dependent on the gradient of the mean streamwise velocity. 


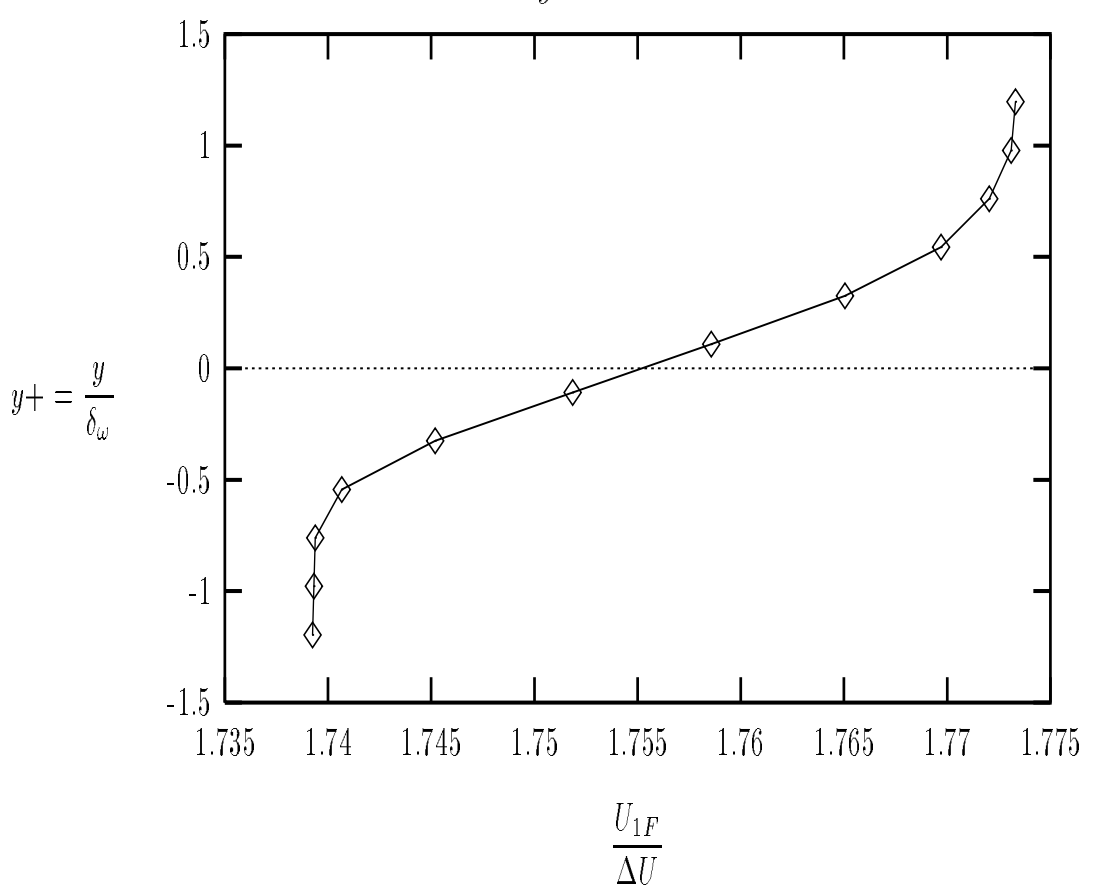

FiguRE 7. Mean streamwise velocity for 7 mode no-feedback simulations.

\subsection{Dynamics as a function of $\alpha$}

Before discussing the dependence of $\alpha$ we will consider behaviour that occurs regardless of the value of $\alpha$. There is an underlying periodic (sinusoidal) behaviour that exists for all modes in the truncation. The frequencies of this behaviour depend on the streamwise wavenumber and are shown in table 1.

\begin{tabular}{cccccccc}
\hline$\left(k_{1}, k_{3}\right)$ & $(10,0)$ & $(20,0)$ & $(30,0)$ & $(40,0)$ & $(50,0)$ & $(60,0)$ & $(70,0)$ \\
Frequency $(\mathrm{Hz})$ & 98.9 & 196.6 & 294.4 & 392.0 & 489.6 & 587.6 & 685.1 \\
\hline
\end{tabular}

Table 1: Fundamental frequency for wavenumber pairs in the seven mode system.

This periodic behaviour is always buried in the time traces of the coefficients, to some scale, regardless of larger time scale events. The frequency increases with larger values of $k_{1}$; since streamwise wavenumbers are mapped from frequency, which vary by less than 
$0.5 \%$ of the values in table 1 , this seems intuitively correct. Other complex behaviour, which will be discussed in further detail, sits on top of the underlying periodicities. Rajaee et al. (1994) obtained similar behaviour in a forced plane mixing layer where the results showed their coefficients exhibiting a complex behaviour on top of underlying periodicities.

The other behaviour that is observed regardless of the value of $\alpha$ is that the mode $(20,0)$ appears to lead the temporal evolution, i.e. the coefficient has a larger magnitude and tends to be the first coefficient to increase or decrease in time. This is consistent with the results discussed in $\S 6$ where this mode was found to be the most linearly unstable. It is also interesting to note that this streamwise wavenumber is associated with energy in the centre of the mixing layer (see Delville et al. 1998). This behaviour is also consistent with the results of Metcalfe et al. (1987) where it was postulated that disturbances from the centre of the mixing layer manifest into the instabilities that cause the flow to exhibit three-dimensionality.
Value of $\alpha$
Behaviour
$\alpha<1.55$
All modes grow unbounded to infinity
$1.55<\alpha<1.75$
Complex behaviour in full space
$1,8<\alpha<2.0$ Intermittent/Complex Periodic behaviour for all modes
$2.05<\alpha<2.45$
Periodic Behaviour
$\alpha>2.5$ Trivial solution

Table 2: Description of dynamics for seven mode no-feedback model.

The typical dynamic behaviour for different values of the bifurcation parameter $\alpha$ is outlined in table 2. For values of $\alpha$ less than 1.55 the system is unstable and grows 
to infinity. In this range of the bifurcation parameter $\alpha$, the term $\Lambda^{2}$ linked to turbulent production, grows at a rate which the dissipation term $\Lambda^{1}$ cannot overcome. While, for $\alpha>2.5$, the behaviour is the opposite. The global attractor for the system is a trivial solution. This means that all the modes decay to zero. Most of the modes decay at a similar rate except for the mode $(20,0)$ which has a much larger decay rate.

For $\alpha$ in the range of $2.05<\alpha<2.45$ the system exhibits a periodic behaviour. Figure 8 displays the real and imaginary parts of the time histories for $\alpha=2.2$ which is indicative of the dynamical behaviour in this range of $\alpha$. For these time history plots of the coefficients, the abscissa is time in seconds. In part a) of this figure, long time evolution of the coefficients is plotted while part b) is a more detailed view of the behaviour over 0.4 second window. The time traces for values of $\alpha$ in this range are best described as two sinusoids superimposed on each other. The first is underlying streamwise wavenumber dependent frequency, while the second varies with $\alpha$ for all modes. Increasing $\alpha$ decreases the amplitude of the second frequency. The mode $(20,0)$ always leads on the secondary frequency acting like it transfers to all the other modes. For streamwise wavenumbers greater than 30 , the amplitude of the oscillations is small, generally an order of magnitude less than the initial condition. While for streamwise wavenumbers less than 30 , the amplitude tends to remain the same order as the initial conditions.

For $\alpha$ in the range of $1.8<\alpha<2.0$ the system exhibited an intermittent periodic behaviour. In this range of $\alpha$ the solutions tend to exhibit a complex secondary period behaviour superimposed on the underlying periodicities. This behaviour would intermittently go through bifurcations in time and pop in and out of the secondary periodicities. Modes $(10,0),(40,0)$ and $(60,0)$ exhibit behaviour which is indicative of two sinusoids while the other modes still exhibit two periodicities although not in as simple of a manner. 

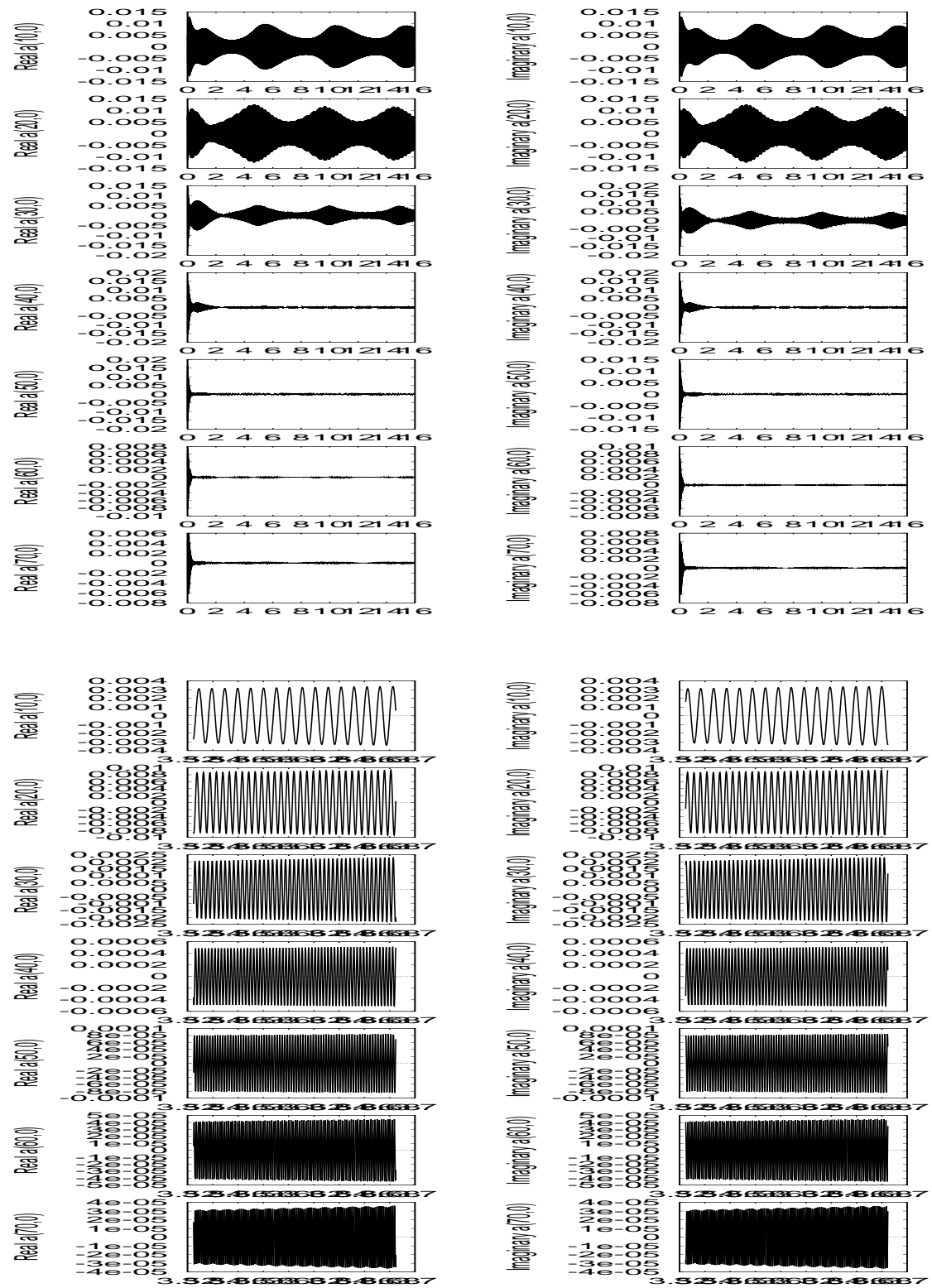

FIGURE $8 . a_{k_{1}, k_{3}}(t) \alpha=2.2$ a) long time evolution b) short time evolution.

For $\alpha$ in the range of $1.55<\alpha<1.75$ the system exhibits a complex behaviour for all modes. In this region the coefficients have oscillations with amplitude on the order of the initial conditions which then grow to larger amplitude, chaotic looking motions. This behaviour is observed for all values of $\alpha$ in this range; however, the larger the value is, 
the longer it takes to exhibit large amplitude behaviour. The large amplitudes observed in this region are order 1 which is significantly larger than the initial conditions shown in table 4 . This can be indicate that $\alpha$ is too small and too little energy is being removed from the system. It is interesting to note that for this range of $\alpha$, the real and imaginary parts exhibit identical behaviour with only a small phase difference.

\subsection{Reconstructed velocity field}

In this section, the instantaneous velocity field will be reconstructed from the results of the simulations discussed in the previous section. The velocity is reconstructed using the inverse Fourier transform of (2.4). For the simulations only involving $k_{3}=0$, discussed in this section, $u_{3}$ is zero (recall from $\S 5.1$ that $\Phi_{3, k_{1}, k_{3}=0}^{(1)}=0$.). Then, this allows for only the reconstruction of an $x, y$ plane.

In the following, only two values of $\alpha$ are studied, 2.2 and 1.85. In each of the plots shown in this section, the flow goes from left to right. The time increment between plots is $4.010^{-04}$ seconds which is five times the minimal resolved time step. The dimensions of the window are $345 \mathrm{~mm}$ in the $x_{1}$ direction and $66 \mathrm{~mm}$ in the $x_{2}$ direction. The figures are plotted in a frame of reference moving at the convection velocity $U_{c}$. This is to say that the velocity plotted is

$$
u\left(x_{2}\right)+U\left(x_{2}\right)-U_{c}
$$

where $U\left(x_{2}\right)$ is the mean streamwise velocity calculated for the particular truncation (see figure 7).

Figure 9 displays the velocity vector plots for $\alpha=2.2$. The simulations yielded essentially periodic solutions for this value of $\alpha$. In the "pseudo" real space plotted here, this is 


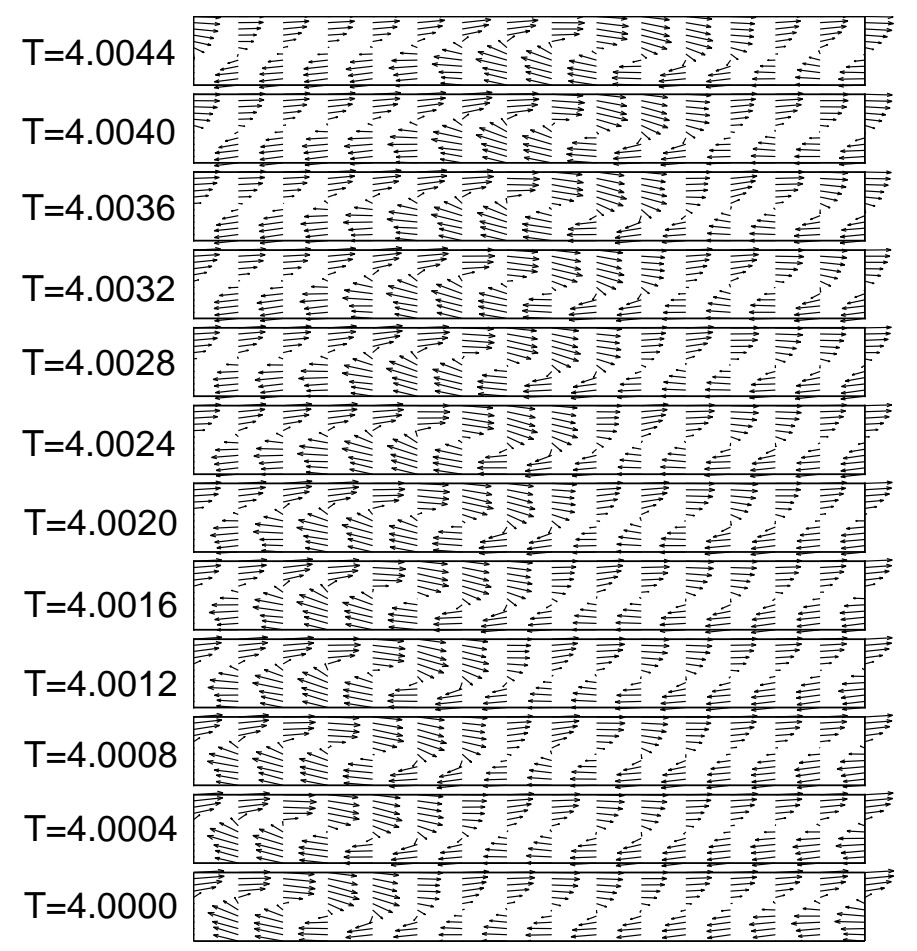

Figure 9. Velocity vector plots for $\alpha=2.2$.

represented by what appear to be spanwise aligned vortices passing through the window. The structures pass through the window at evenly spaced intervals without interacting with each other. This type of solution is consistent with the results of Metcalfe et al. (1987) who observed roll-up without pairing in the absence of sub-harmonic excitation. These results are also similar to those of Rajaee et al. (1994) where the mixing layer was forced and where periodic solutions with structures being convected through the resolved window were found. The average length of these structures, in the $x_{1}$ direction, is about $215 \mathrm{~mm}$. While in the $x_{2}$ direction they have a scale equivalent to the vorticity thickness. 


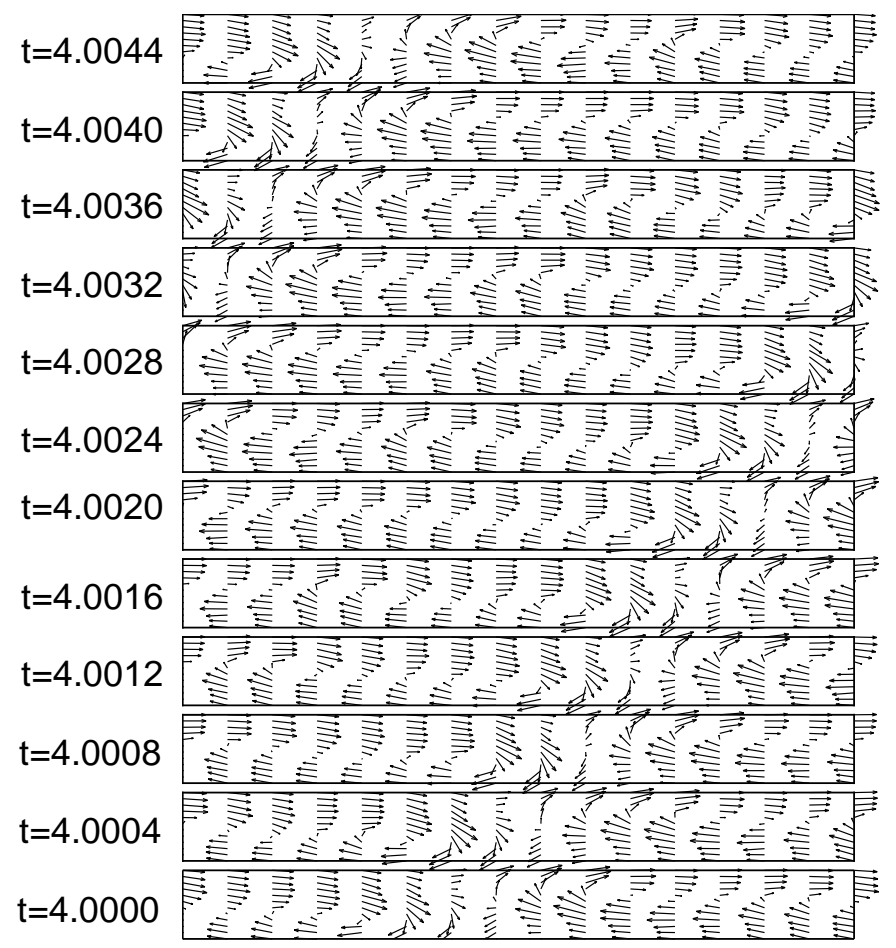

FIGURE 10. Velocity vector plots for $\alpha=1.85$.

Figure 10 shows the velocity vector plots for $\alpha=1.85$. These plots exhibit more active behaviour than the plots for $\alpha=2.2$. There appears to be similar structures to $\alpha=2.2$ passing through the windows along with other events. The other major event can be observed in the first window $(t=4 \mathrm{~s})$. This structure is much longer in the streamwise direction and appears to have two cores. This is how a pairing event would appear plotted with velocity vectors although this organization does not seem to have the energy wrap around as would be truly indicative of a pairing event. The scenario of these two events passing through the window has a regular period. 


\subsection{Reconstructed energy profiles}

Once the velocity fields have been reconstructed, the turbulent kinetic energy profiles can be reconstructed by averaging the time series data. These profiles have also been calculated directly from the first POD modes using the equations:

$$
\begin{aligned}
{\overline{u_{i}^{\prime} u_{j}^{\prime}}}^{(1)}\left(x_{2}\right) & =\iint_{-\infty}^{+\infty} \Psi_{i j}^{(1)}\left(x_{2}, x_{2} ; k_{1}, k_{3}\right) \mathrm{d} k_{1} \mathrm{~d} k_{3} \\
& =\iint_{-\infty}^{+\infty} \lambda_{k_{1}, k_{3}}^{(1)} \Phi_{i, k_{1}, k_{3}}^{(1)} \Phi_{j, k_{1}, k_{3}}^{(1) *} \mathrm{~d} k_{1} \mathrm{~d} k_{3}
\end{aligned}
$$

As long as significant averaging was used, the two methods yielded similar results.

Figure 11 is a plot of the measured components of the turbulent kinetic energy profiles. The values in this plot are obtained with $(7.1 a)$ where the integral in wavenumber space is from $k_{1}=0$ to $70 \times \delta k_{1}$ exp for $k_{3}=0$. In this plot one can see that the kinetic energy from $v$ is dominant. This is consistent with the discussion on the spectral tensor in Delville et al. (1998) where it was shown that the energy associated with $v$ is dominant at $k_{3}=0$ while the energy associated with $u$ is more broad band and spread out over several values of $k_{3}$.

Figures 12 through 14 are plots of the Turbulent kinetic energy profiles for $\alpha=$ 2.2, 1.85 and 1.7 , respectively. All of these plots show the streamwise component of the Reynolds stress being dominant with a $x_{2}$ distribution similar to the measured value (figure 11). The model represents quite well the streamwise component of Turbulent kinetic energy. This should be expected because in the application of the POD, the Reynolds stress is very well reconstructed by the first POD mode. For the values of $\alpha$ studied, the $u v$ profile is approximately one-third of the streamwise Turbulent kinetic energy profiles which is in good agreement with the experimental data. As with the vv 


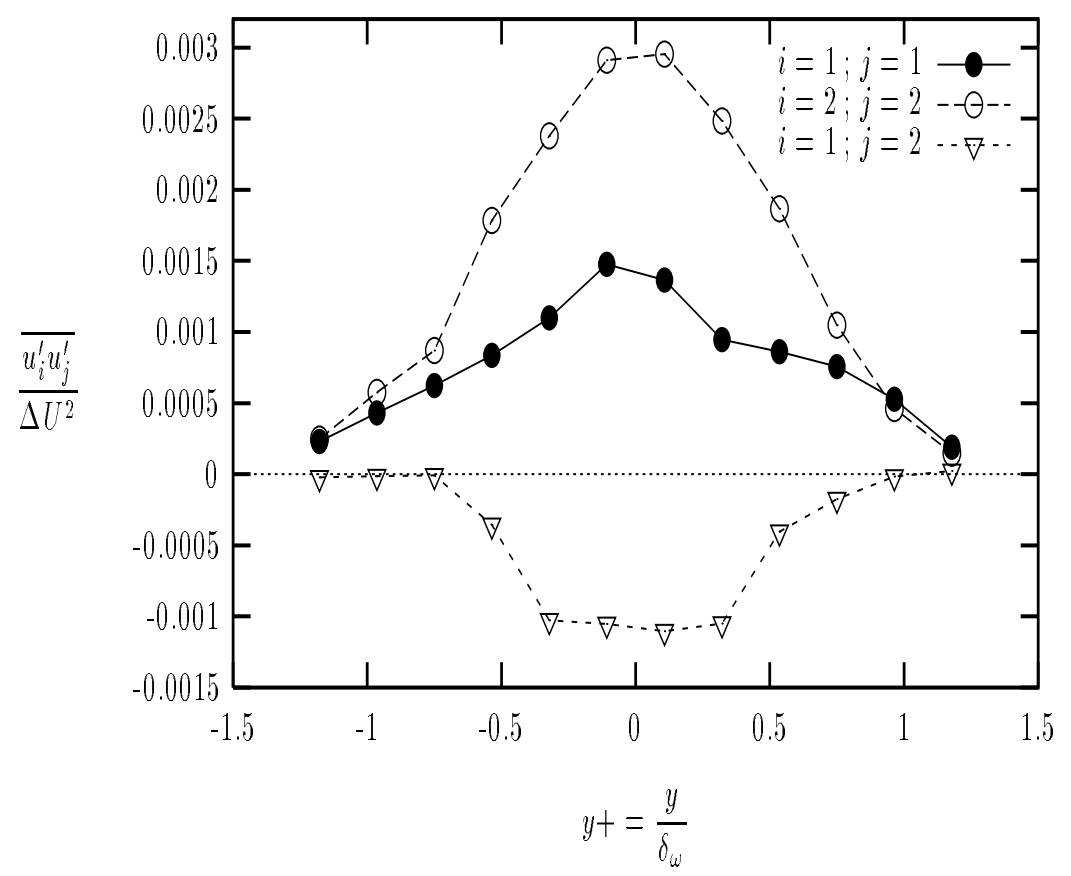

FIGURE 11. Turbulent kinetic energy profiles integrating from measured spectra for wavenumbers kept in the truncation.

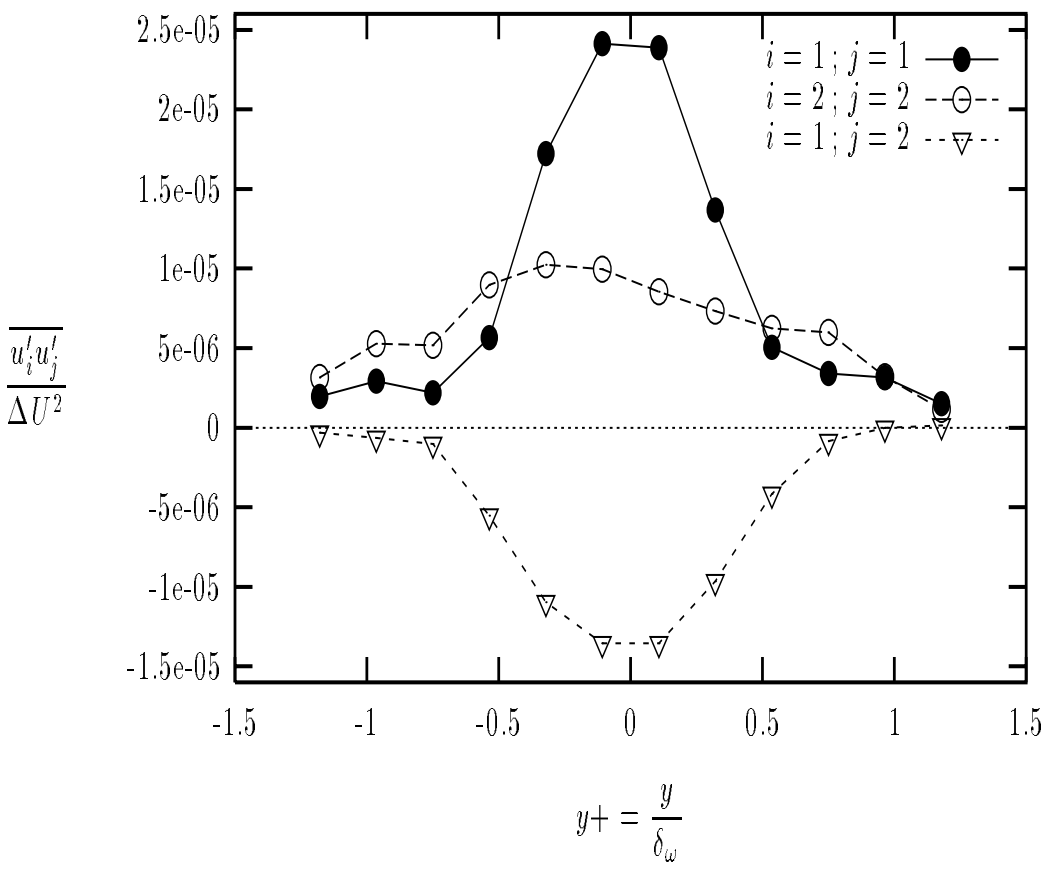

Figure 12. Turbulent kinetic energy profiles $(\alpha=2.2)$. 


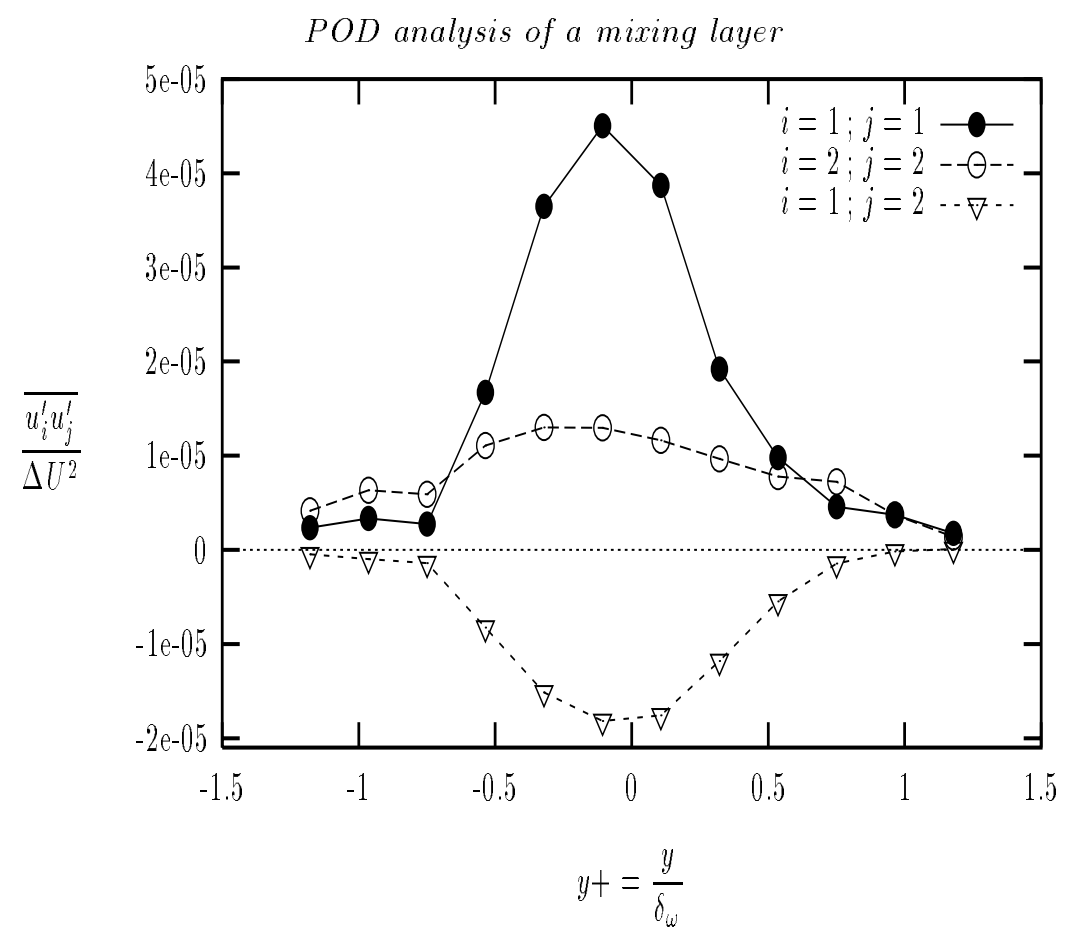

FiguRE 13. Turbulent kinetic energy profiles $(\alpha=1.85)$.

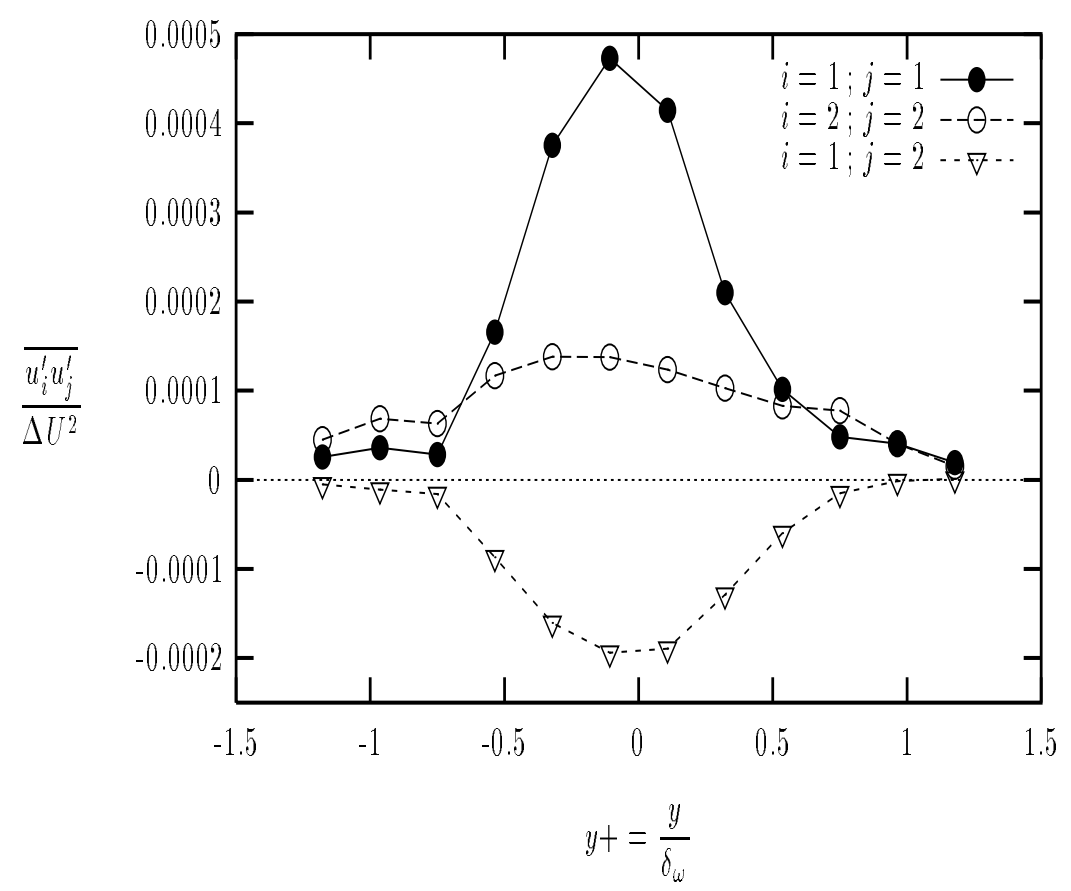

Figure 14. Turbulent kinetic energy profiles $(\alpha=1.7)$. 
component, the model is not able to represent correctly the Reynolds stress for all values of $\alpha$ (the reason for this discrepancy is discussed in the next paragraph).

When figures 12 through 14 are compared with figure 11, the most striking feature is the swapping of dominance between the $u$ profile and the $v$ profile. This is caused by the coefficients for the first two modes, $a_{10,0}$ and $a_{20,0}$, being dominant in amplitude for the simulations. It was shown in the discussion of the spectra in Delville et al. (1998) that streamwise wavenumbers less than $5.8 \mathrm{~m}^{-1}$ are associated with $u$ energy at the centre of the mixing layer. When calculating the profiles in figures 12 through 14 the two first modes become the dominant terms in the integration over streamwise wavenumber, thus making the $u$ energy profile dominant. It is as if the model compensated for the distribution of energy of the average quantities by altering the magnitudes of the instantaneous coefficients to arrive at the correct energy distribution.

\subsection{Spectra of reconstructed velocity}

Figures 15 and 16 display autospectra, calculated from the reconstructed velocities discussed above for the $u$ component and the $v$ component of velocity, respectively. In these plots the $y$ axis has units of $m^{2} / s^{2} / H z$ while the $x$ axis is a non-dimensional frequency, $f \delta_{\omega} / U_{c}$. Only the spectra from half of the mixing layer are included here because the upper and lower halves demonstrated the same characteristics consistent with the measured spectra presented in Delville et al. (1998). Only spectra for $\alpha=1.85$ are shown because the behaviour described below is representative of results regardless of the value of $\alpha$.

In figures 15 , the $x_{2}$ distribution of energy that was discussed in the experimental 

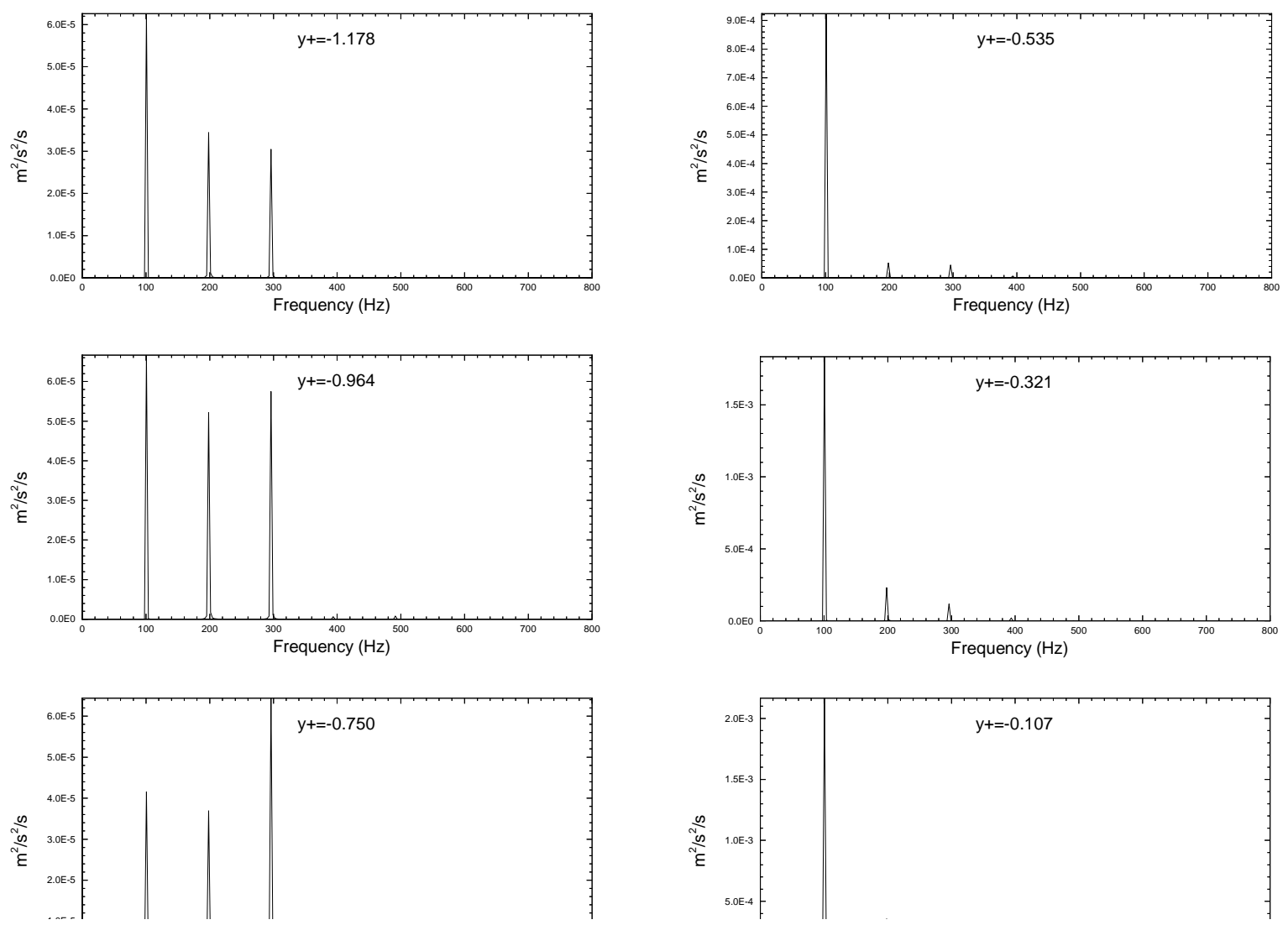

FIgURE 15. Autospectra $u_{1}$ component $\alpha=1.85$.

section on spectra (see Delville et al. 1998) is clearly pronounced. For the outer part of the mixing layer, $x_{2} / \delta_{\omega}=-0.75$ through -1.178 , the energy distribution is similar to that for the $v$ component and contains information near the dominant Strouhal number. In the centre of the mixing layer, the energy is contained at lower frequencies.

For the $v$ component of velocity (figure 16), a similar energy distribution in frequency regardless of the $x_{2}$ location is obtained, while the amplitudes reach a maximum near the centre of the mixing layer. The frequencies observed here correspond closely to the experimentally observed values. 

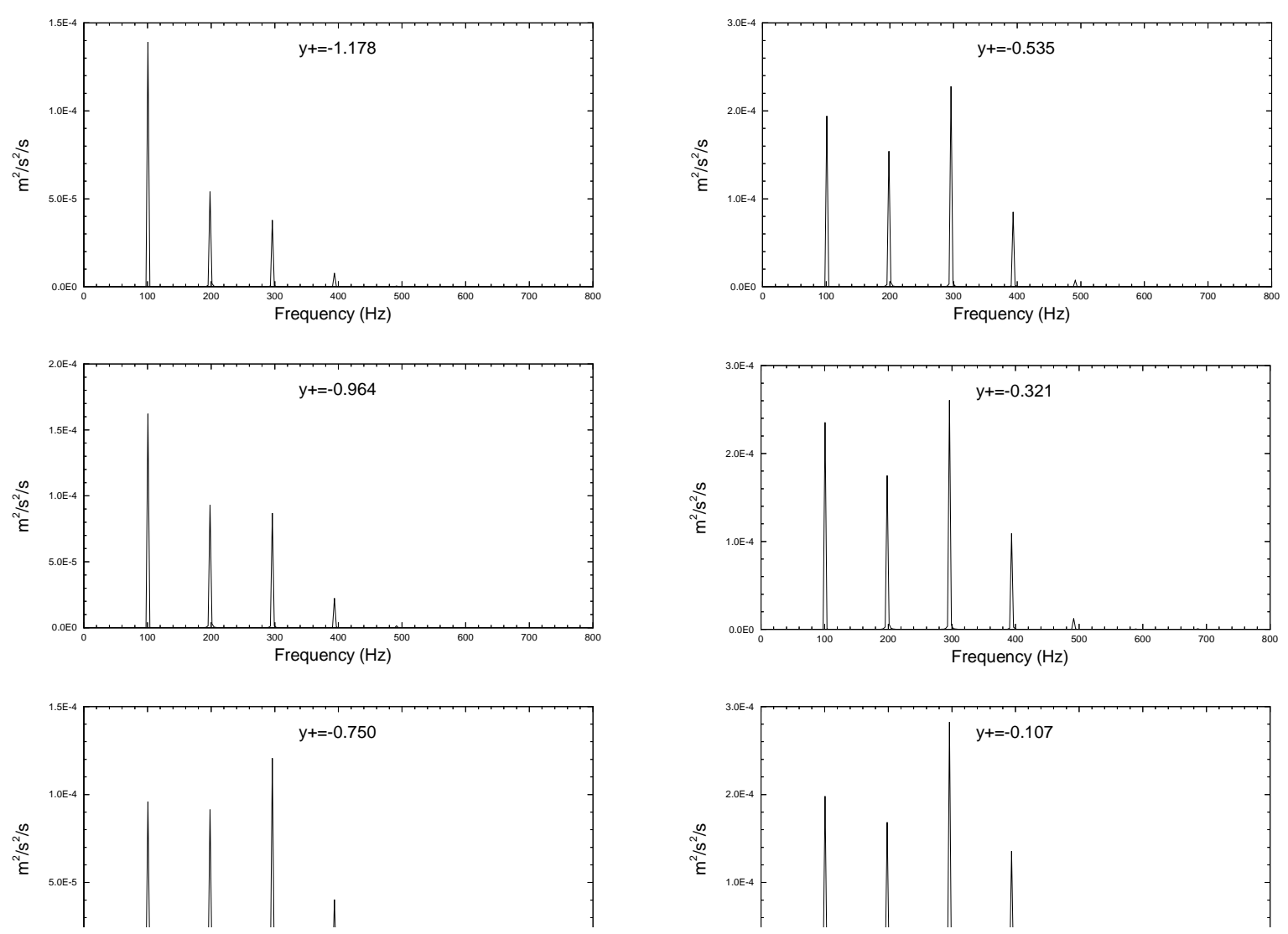

Figure 16. Autospectra $u_{2}$ component $\alpha=1.85$.

The behaviour described here for the $x_{2}$ distribution of spectra mimics well the experimental behaviour presented in Delville et al. (1998). It is very encouraging that our severely truncated model has the correct trends in the spectral distributions.

\subsection{7 mode model filter}

In this section, the solution of (4.1), written for the filter case, will be discussed for the seven mode system retained in $\S 7.1$. With the method of closure of (3.7), the mean velocity is split into a steady part that is held constant for all time and a time dependent part that manifests itself as a cubic term in the dynamical equations. The introduction 


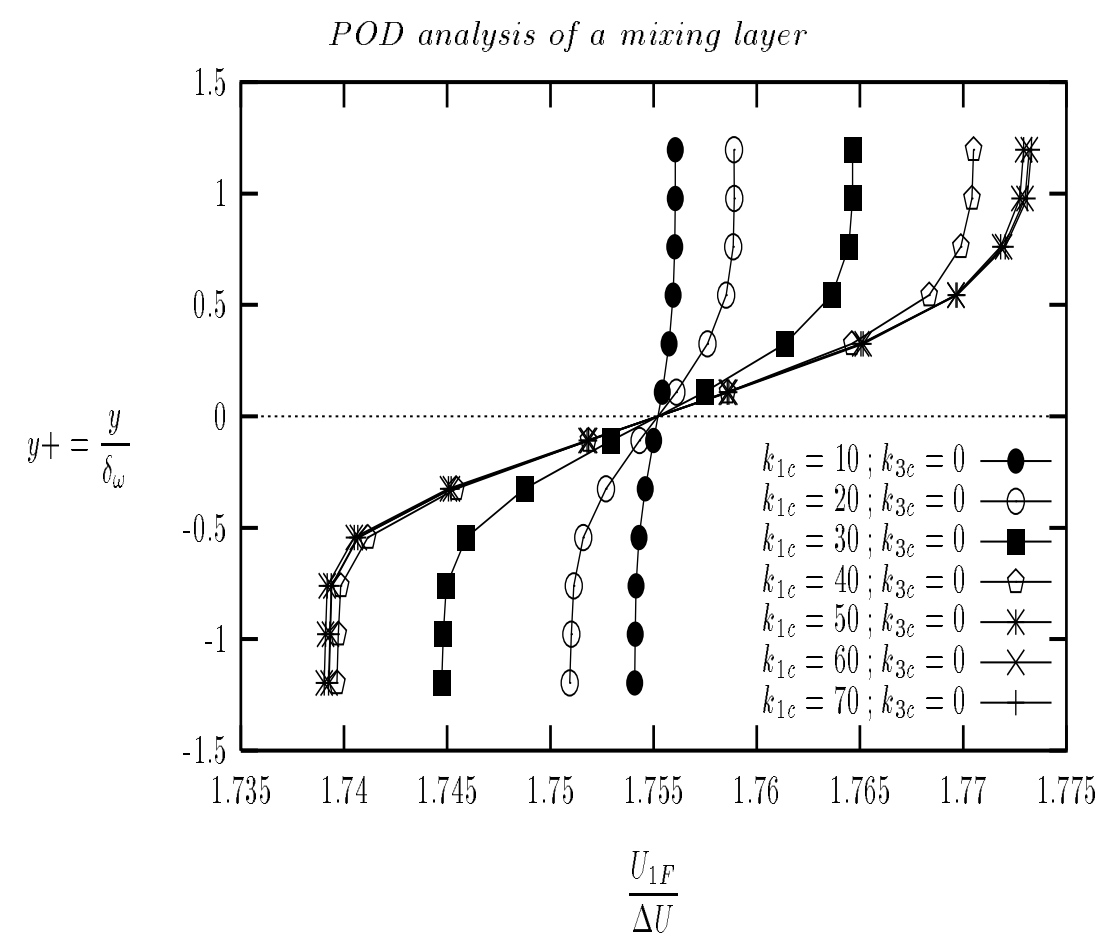

FiguRE 17. Mean streamwise velocity profiles for different filter values.

of this filtering technique discussed in $\S 3$, allows time dependent interaction between the coherent structures and mean velocities. This technique will also allow for the scaling of the production term. The need for this will become apparent when truncations involving $k_{3} \neq 0$ are discussed in $\S 8$.

Figure 17 shows $U_{1 F}$ estimated from (3.6) for different values of $k_{1 C}$ and $k_{3 C}$. For values of $k_{1 C}=50$ or 60 , the mean velocity profiles are essentially the same as the one used in the no-feedback case $\left(k_{1 C}=70\right.$ and $k_{3 C}=0$ in figure 17$)$. Then, as one should expect, the lower the filter value $k_{1 C}$ is, the smaller the gradient in the mean velocity profile is. 
7.6.1. Filter cutoff $=(50,0)$ or $(40,0)$

Since the simulations of the no-feedback yielded reasonable results, it seemed like a natural first step to start with $k_{1 C}=50$. This allows for some contribution to the cubic term without much loss in mean streamwise velocity gradient. Upon further examination of figure 17 , one notices that when $k_{1 C}=40, U_{1 F}$ is relatively close to the profile obtained for $k_{1 C}=50$; and in fact these two values of $k_{1 C}$ yielded similar dynamics in numerical integrations. Since the mean streamwise velocity profile is essentially the same, the turbulent production terms in (4.1) would have the same order of magnitude; yet the introduction of the cubic term should act as a feedback between turbulent and mean streamwise velocities.

Initial results from the numerical simulations showed that the introduction of the cubic term had a stabilizing effect on the system $\dagger$. For values of $\alpha>1.55$ the behaviour is the same as for the no-feedback case and is still characterized by the descriptions in table 2. Now, however for values of $\alpha<1.55$ the system no longer exhibits the unbounded growth mentioned in the discussion of the no-feedback case. For values of $\alpha$ in this range the system now exhibits a complex behaviour in full space on top of the underlying periodicities, much the same as the region between $1.8>\alpha>1.55$ for the no-feedback case. For example, when $\alpha=0.5$, the complex behaviour is similar to that of the no-feedback simulations although the mean square amplitudes of the coefficients have increased even more. These characteristics were observed whether $k_{1 C}=50$ or $k_{1 C}=40$.

$\uparrow$ We recall that in the context of dynamical system theory, the cubic terms may have stabilizing effects when their contribution is the opposite of the linear term contribution. 
7.6.2. Other values for cutoff wavenumber

For values of $k_{1 C}$ smaller than 40 , the system took on different characteristics which indicate the importance of having the correct level of production. For $k_{1 \mathrm{C}}=20$ and $k_{1 \mathrm{C}}=$ 30 the dynamical behaviour is similar. The systems yielded trivial solutions except for small values of $\alpha$. For these small values, the dynamics were very similar to the complex behaviour discussed for the no-feedback model although the mean square amplitude of the coefficients were more reasonable when compared to the experimental values.

As one would expect, the smaller the value of $k_{1 C}$, the more closely the system mimics the limiting case examined in the section on linear stability. Although as long as there was some contribution of the production term, the systems never reached the region of unbounded growth and they always appeared to be decaying systems. The reason for this is again the lack of linear growth as stated previously. It is also noticed that as $U_{1 F}$ is decreased the production term is reduced in magnitude. This allows for the complex secondary behaviour to occur with the smaller amplitude of the coefficients.

\section{Truncations for $k_{3} \neq 0$}

In this section, simulations of both the no-feedback and the filtered equations will be presented for truncations that included modes with non-zero spanwise wavenumbers. It was observed in the results of truncations for $k_{3}=0$ that some of the instantaneous and statistical features have been well represented. The extension was performed in an attempt to mimic the full field flow dynamics and see if the dynamics observed in the smaller truncations still existed. 
The specific truncation studied here is an extension of a five mode model $\left(k_{1}=\right.$ $\left.10,20,30,40,50 ; k_{3}=0\right)$ discussed in Ukeiley (1995). In brief, the smaller system exhibited essentially the same dynamics as the seven mode system and allowed for inclusion of non-zero spanwise wavenumbers without the system becoming too large. The extension discussed in this section includes $k_{3}=1,2$ and 3 . This involves the solution of equations for 38 modes. Table 5 in appendix $\mathrm{C}$ shows the wavenumber pairs and the initial conditions.

For this truncation, the streamwise domain was kept the same as before. The spanwise domain $L_{3}$ is approximately $0.27 \mathrm{~m}$. The plots of $R_{i j}$ in Delville et al. (1998) have a domain size that is large enough to allow the correlations to decay.

\subsection{No-feedback relationship}

Figure 18 displays the mean profile used for the simulations discussed in this section. It has an observably larger gradient than those used for the $k_{3}=0$ simulations, as shown in figure 17. This larger gradient results in an increase in the production term allowing more energy into the system.

\subsubsection{Dynamics as a function of $\alpha$}

Table 3 summarizes the dynamics of the system of equations as a function of $\alpha$. The values of $\alpha$ where interesting dynamics occur are much higher than with the seven mode model. However, with the 38 mode model, the same underlying frequencies, as a function of $k_{1}$ (see table 1 ), were observed regardless of the value of $k_{3}$. With the inclusion of non-zero spanwise wavenumbers, the model now includes three modes involving $k_{1}=0$. 


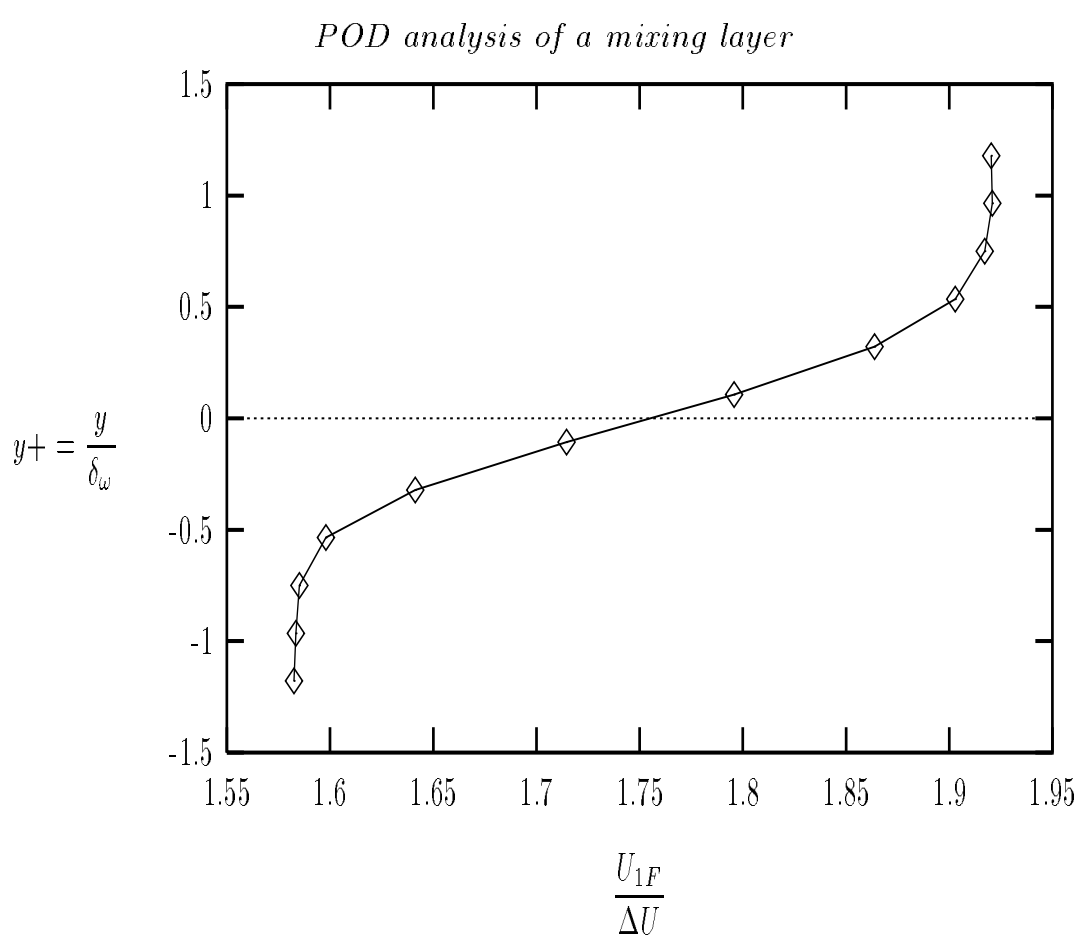

FIGURE 18. Mean streamwise velocity profiles for 38 mode model no-feedback case.

For these modes there was no underlying frequency, causing the events to seem more intermittent than periodic. In the figures showing the time histories of the coefficients only positive values of $k_{1}$ are shown. The negative values of $k_{1}$ exhibited similar dynamics to their positive counterparts; thus their graphical representation has been omitted.

As with the seven mode no-feedback simulations there is a cutoff value for $\alpha$ below which the solutions to the equations grow unbounded. For the 38 mode simulations this value was $\alpha=36.5$. With $\alpha$ greater than 65 the viscous dissipation term is dominant, and a trivial solution is the global attractor. 
Value of $\alpha$

$\alpha<36.5$

$36.5<\alpha<39$

$39<\alpha<45.5$

$45.5<\alpha<47$

$47<\alpha<52.6$

$52.6<\alpha<57$

$57<\alpha<65$

$\alpha>65$
Behaviour

All modes grow unbounded to infinity

Unstable solutions switching between

unbounded growth and complex behaviour

with low-order amplitude coefficients

Complex behaviour in full space

Complex behaviour for $k_{3}=0$

Intermittent behaviour for $k_{3} \neq 0$

System blows up

Complex behaviour for $k_{3}=0$

Trivial solution for $k_{3} \neq 0$

Intermittent/periodic solution $k_{3}=0$

Trivial solution for $k_{3} \neq 0$

Trivial solution for all space

Table 3: Description of dynamics for 38 mode no-feedback model.

When $36.5>\alpha>39$, the system switched between complex behaviour and unbounded growth with small changes in $\alpha$. In this range, when the solutions did not grow unbounded, all the modes exhibited a complex 'random' solution over the underlying periodicity. The behaviour of the system for $\alpha$ between 39 and 45.5 is similar to the one obtained for the values of $\alpha$ that did not grow unbounded in the previous range. For these values of $\alpha$ when $k_{3}=0$, the solutions exhibit very similar dynamics. However, when $k_{3} \neq 0$, the solutions switch between behaviour, similar to the one obtained for the modes $k_{3}=0$ and an intermittent type of behaviour with the coefficients being zero 
between events. Also as $\alpha$ approaches 47 the amplitude of the coefficients for $k_{3}=0$ grow until in the region of $\alpha$ between 47 and 52.6 they cause the system to become unstable.

The dynamics for $\alpha$ between 52.6 and 65 follow trends similar to the seven mode model for $\alpha$ between 2.05 and 2.45, especially in the observed intermittent behaviour. For all the modes with $k_{3} \neq 0$, a trivial solution was obtained, while for $k_{3}=0$, all modes in $k_{1}$ exhibited active dynamics. These modes evolved through periodic solutions with the underlying periodicity remaining constant and the secondary periods becoming larger and larger. As with the other simulations, mode $(20,0)$ is controlling the dynamical behaviour of the system. This mode grows in amplitude dragging the mode $(40,0)$ with it. Then these two modes distribute their energy to the rest of the system. These results are interesting because they imply that the flow is relaxing to a two-dimensional state. For these values of $\alpha$, the system is being damped so much that any variations in the streamwise direction are not able to arise. However, this is the only range of $\alpha$ that has realistic amplitudes for the coefficients. This implies that when the filtering technique is applied to the truncations involving $k_{3} \neq 0$, it should be possible to obtain threedimensional dynamics at the correct coefficient amplitudes.

\subsubsection{Representation in physical space}

Figures 19 and 20 display the turbulent kinetic energy profiles for $\alpha=39$ and 45 , respectively. As with the seven mode dynamical system, these systems exhibit distributions in the $x_{2}$ direction similar to the experimental distributions. For both of these profiles the streamwise turbulent kinetic energy is much higher than expected. For $\alpha=45$ it is even greater than the experimental values. This result, although not very physical, is due do the high magnitudes of the coefficients when compared to the initial conditions. Using 


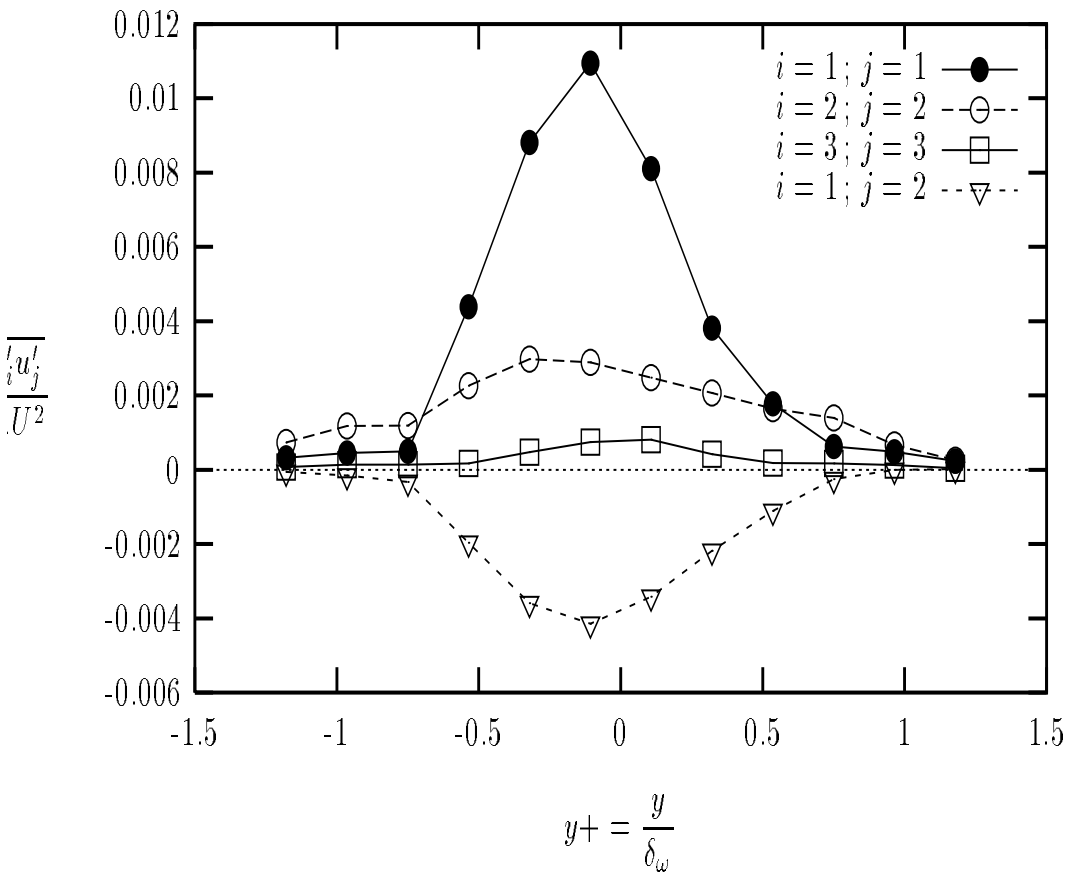

Figure 19. Turbulent kinetic energy profiles for $\alpha=39$ (38 mode model).

the mean streamwise velocity profile shown in figure 18 causes too much production and the system cannot obtain a three-dimensional solution with realistic values for the coefficients. The amplitude of the Reynolds stress were always found to be significantly larger than those of the experimental values. This is a result of too much production causing the amplitudes to be too large and shows why there is a need to scale the linear growth term.

\subsection{8 mode model filter relationship}

The results discussed for the 38 mode no-feedback model suggests that using the filter technique would prove useful in scaling the production term and obtaining coefficients with a more realistic amplitude. The effects of the filter were not the same as for the trun- 


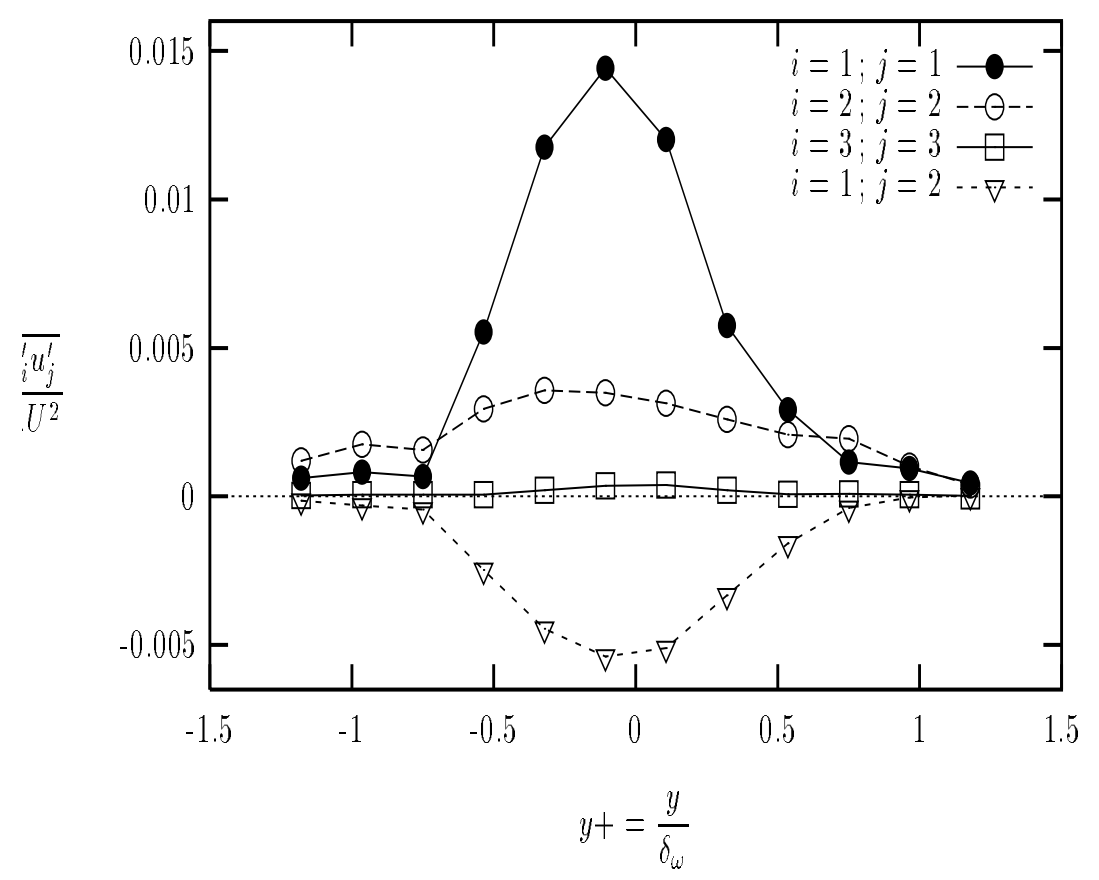

Figure 20. Turbulent kinetic energy profiles for $\alpha=45$ (38 mode model).

cation involving only $k_{3}=0$, although the desired results were achieved. The differences will be discussed in the next paragraphs.

Several values of filter setting $\left(k_{1 C} ; k_{3 C}\right)$ have been evaluated in this study. For values of the filter that extremely reduced the mean streamwise velocities gradient, the system exhibited a behaviour where the coefficients were always decreasing (for example in Ukeiley 1995 simulations were presented where all of the mean velocity was modeled in the feedback term). For values where the filter did not alter the mean streamwise velocities gradient significantly, the system behaved similarly to the no-feedback results discussed in the previous section as was expected. Finally, a setting of $k_{1 C}=40$ and $k_{3 C}=2$ will be focus of the following discussion. 


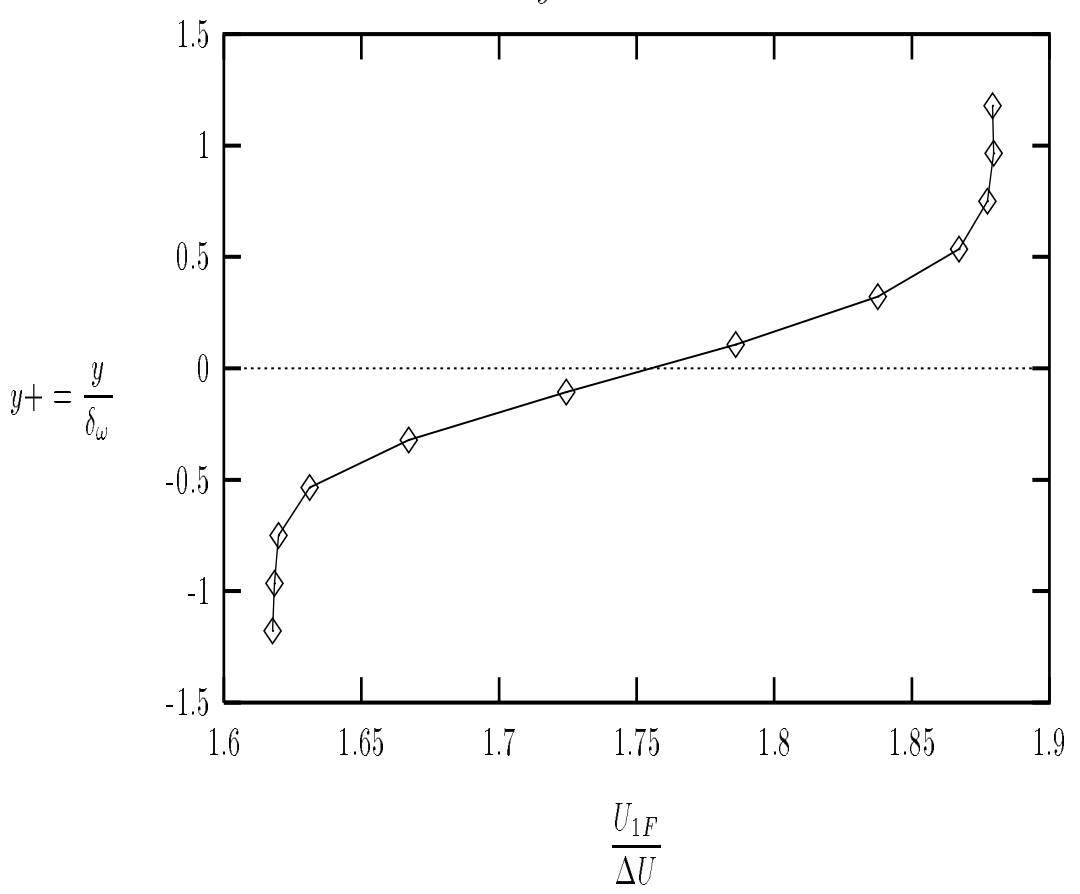

FigurE 21. Mean streamwise velocity profiles for filter setting of $(40,2)$ (38 mode model).

\subsubsection{Filter cutoff $(40,2)$}

Figure 21 shows a profile of $U_{1 F}$ calculated with a filter setting of $(40,2)$. It has a noticeably smaller gradient than that of the unfiltered case (see figure 18) resulting in a reduction of the production term in (4.1). The smaller production term should limit the energy input into the model resulting in coefficients with smaller amplitudes.

The dynamics exhibited as a function of $\alpha$ were simpler than for the no-feedback case described in the previous section. For $\alpha<35$ the system was unstable and grew to infinity. There was a small range, $39>\alpha>35$, where the system exhibited active dynamics for all modes. For higher values of $\alpha$ the system exhibited the same behaviour as in the no-feedback simulations.

Figure 22 displays the time traces for $\alpha=36.65$. This plot displays a short time 


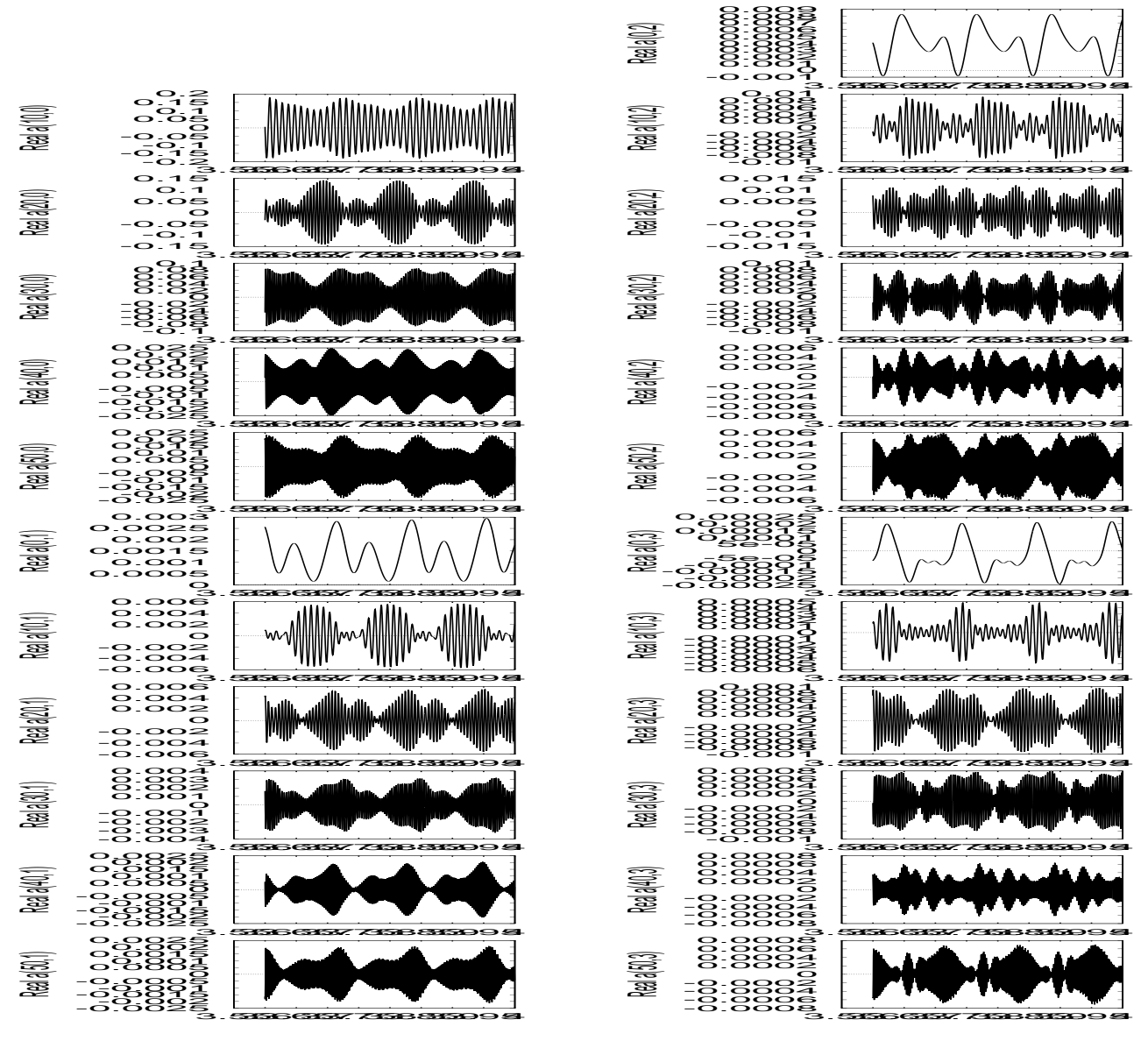

FIGURE 22. Real part $a_{k_{1}, k_{3}}(t) \alpha=36.65$ for a filter setting of $(40,2)$ (38 mode system) short time evolution.

evolution of the coefficients. In this figure, all modes exhibit the underlying periodicities, with a more complex second periodic behaviour sitting on top. The mean square amplitudes of the coefficients are also much closer to the POD eigenvalues than those from the no-feedback case. Hence this is an appropriate filter setting and value of $\alpha$ for examining the reconstructed flow field.

Figure 23 displays the turbulent kinetic energy profiles for $\alpha=36.65$. The behaviour in the $x_{2}$ direction is the same as has been observed in the no-feedback case. However the amplitudes are significantly smaller than those for the no-feedback case. The amplitudes 


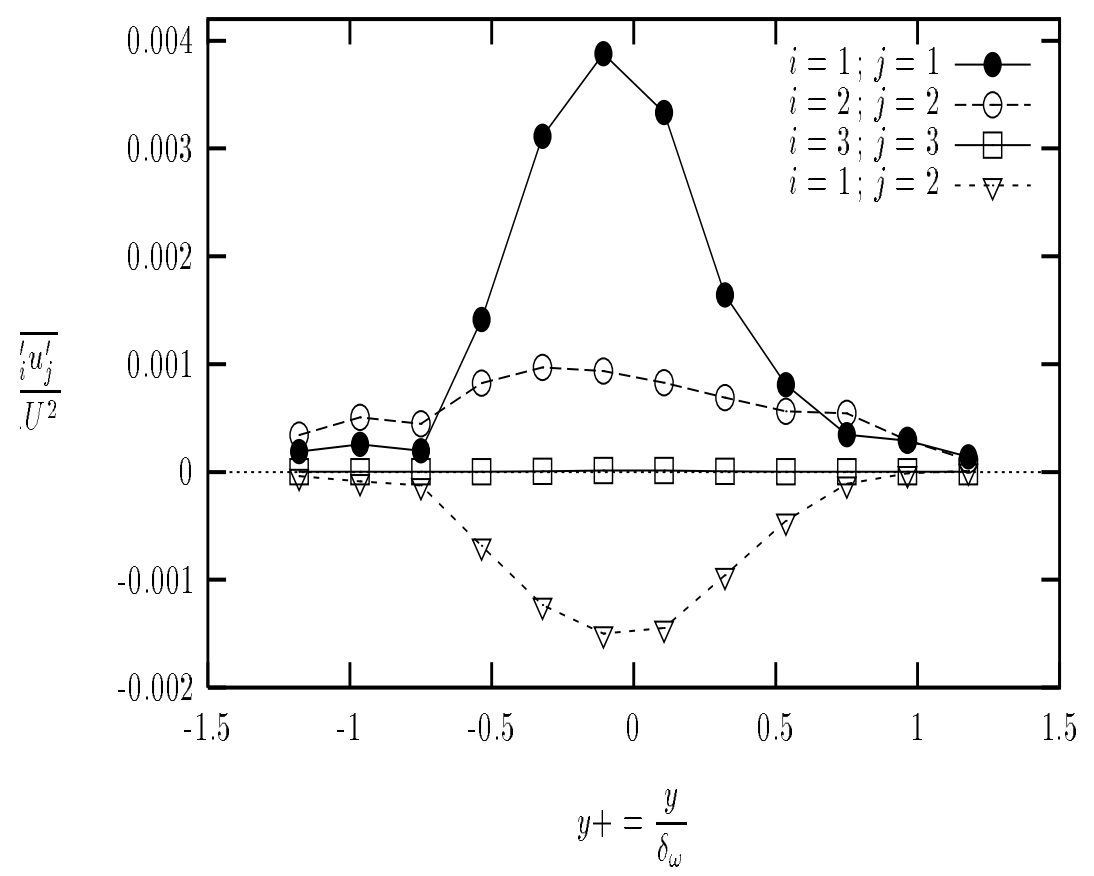

Figure 23. Turbulent kinetic energy profiles for $\alpha=36.65$ with a cutoff filter of $(40,2)(38$ mode model).

are now of the same order as those obtained from integrating the measured spectra over the first POD modes contribution from the wavenumbers kept in the model. The ratio of the $u_{1}$ component to the $u_{2}$ component is now comparable to original experimental values shown in Delville et al. (1998).

Figures 24 and 25 plot the $u_{1}$ and $u_{2}$ autospectra for the case discussed above. The axis on these plots have the same units as for figures 15 and 16 . The $u_{3}$ component is not shown because its magnitude was quite small as evidenced by the amplitude of the Reynolds stress in figure 23. As with the seven mode system ( $\$ 7.5)$, only values from the bottom half of the mixing layer are shown due to the symmetric nature of the flow. These plots exhibit qualitatively the same behaviour as was discussed with the seven mode system. The frequency content of the $u_{2}$ component does not change as a function 

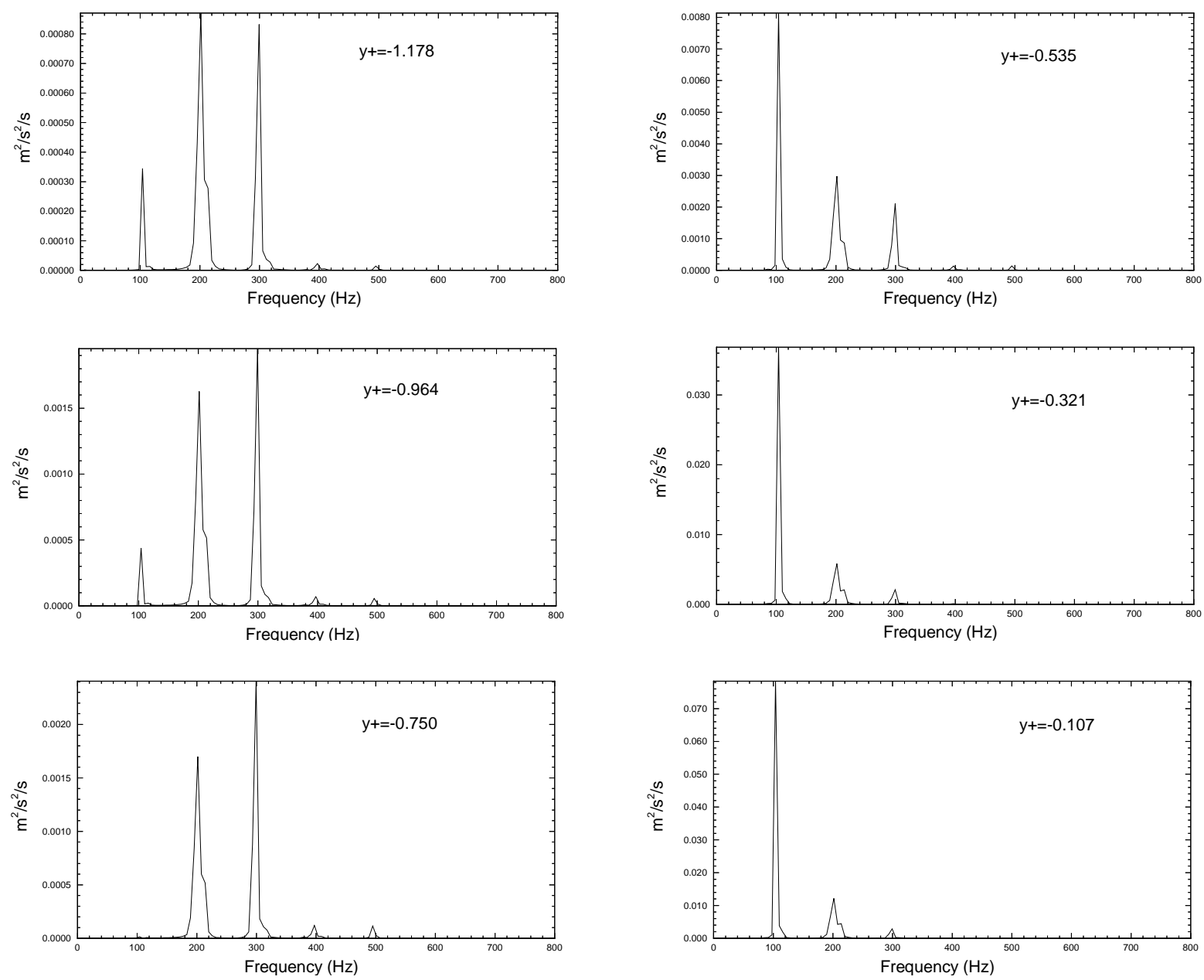

FigURE 24. Autospectra $u_{1}$ component $\alpha=36.65$.

of the $x_{2}$ location, and the amplitude of the spectral peaks gets larger near the centre. The $u_{1}$ component has a shift in the frequency between the inner and outer parts of the mixing layer: the outer region has the same frequency content as the $u_{2}$ component and the inner part has a dominant frequency of approximately one-half the outer. The spectral peaks are now noticeably broader than those shown in figures 15 and 16 because more modes have been included in the system, each containing its own spectral content. 

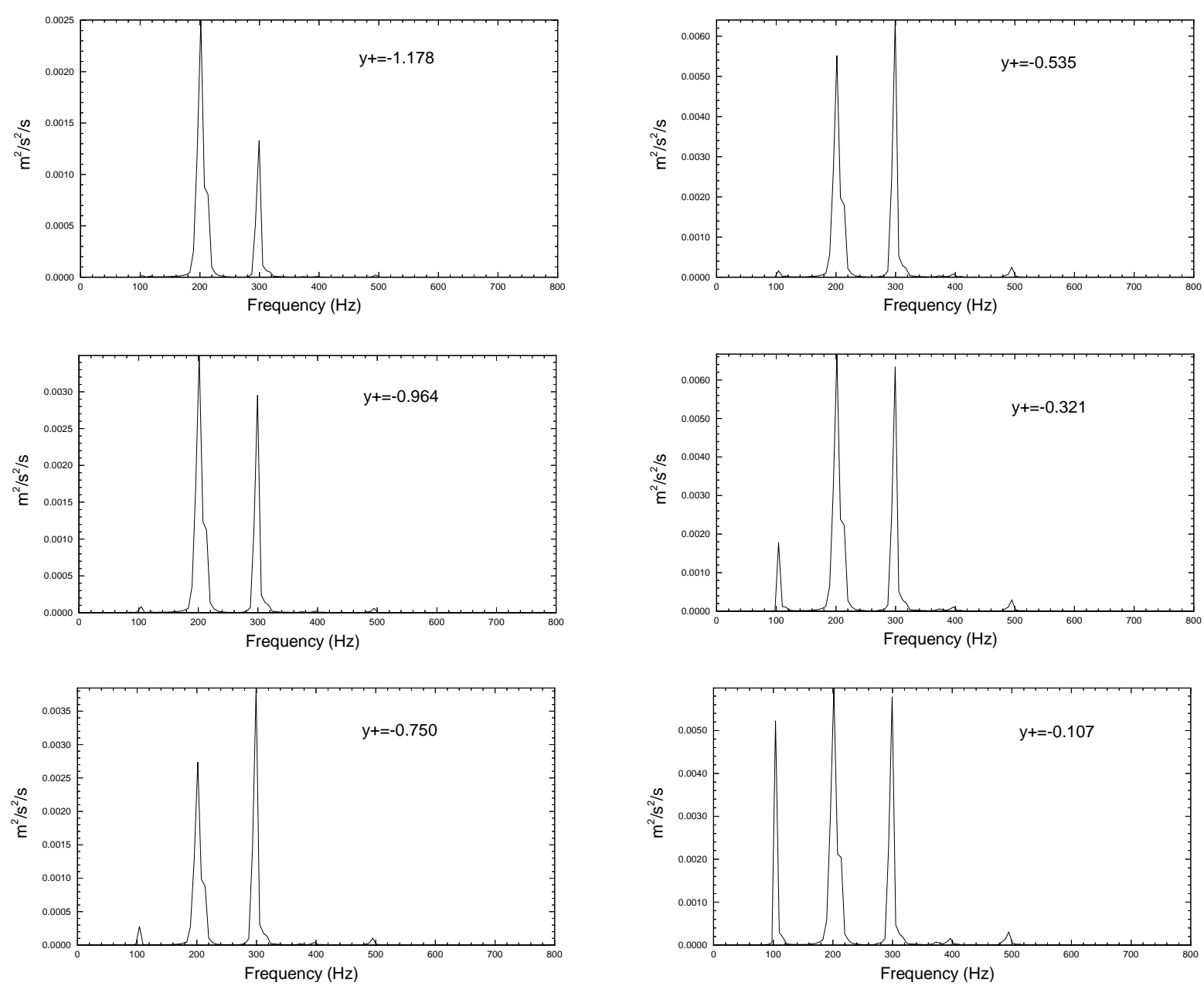

FigURE 25. Autospectra $u_{2}$ component $\alpha=36.65$.

Figures 26 and 27 show plots, determined at $t=3.6$ seconds, of the total vorticity $\dagger$ for the $x_{1}, x_{2}$ and $x_{2}, x_{3}$ planes, respectively. It should be noted that the vorticity plots are utilized here to analyse the reconstructed field instead of velocity vectors due to the complex nature of three-dimensional velocity fields. In figure 26 , a slice of the flow at $x_{3}=0$ is displayed. In this figure, there is strong evidence of several spanwise vortices, e.g. the two contour peaks at locations $\mathrm{B}$ and $\mathrm{F}$ along with the two in the other half of

$$
\dagger\left(\left|\omega_{1}\right|+\left|\omega_{2}\right|+\left|\omega_{3}\right|\right)
$$


the domain. Figure 27 displays the $x_{2}, x_{3}$ plane contours of the vorticity at the locations marked on figure 26. In this figure, there is evidence, between the two spanwise vortices, of streamwise aligned vortices in $x_{1}$. These are represented by the contour lines at locations $\mathrm{D}$ and $\mathrm{E}$ which lie between the spanwise vortex centres at locations $\mathrm{B}$ and $\mathrm{F}$. This result is in good agreement with previous studies of the mixing layer (Metcalfe et al. 1987), where there is strong evidence that spanwise vortex tubes are connected by streamwise aligned vorticity. The average ratio of the spanwise distance between structures to that of the streamwise structures was approximately 0.7 . This is in good agreement with the results of Pierrehumbert \& Widnall (1982) where the most unstable wavelength was shown to be approximately $\frac{2}{3}$.

\section{Conclusions}

In this work, a low-order dynamical system model has been presented that allows to represent some of the properties known to exist in the turbulent mixing layer. The results of the simulations mimic the essential physics as determined by comparisons to actual measurements. It is quite remarkable considering the fact that the model only consists of less than 80 equations. The fact that the system exhibits the correct essential physics makes it a likely candidate to develop control strategies for the mixing layer such as those proposed for the dynamical systems model of the near wall region by Berkooz (1992)

To obtain the ordinary differential equations of the low-order dynamical system, a Galerkin projection of the Navier-Stokes equations on the POD eigenfunctions of Delville et al. (1998) was performed. The first simulation of these equations show clearly the im- 


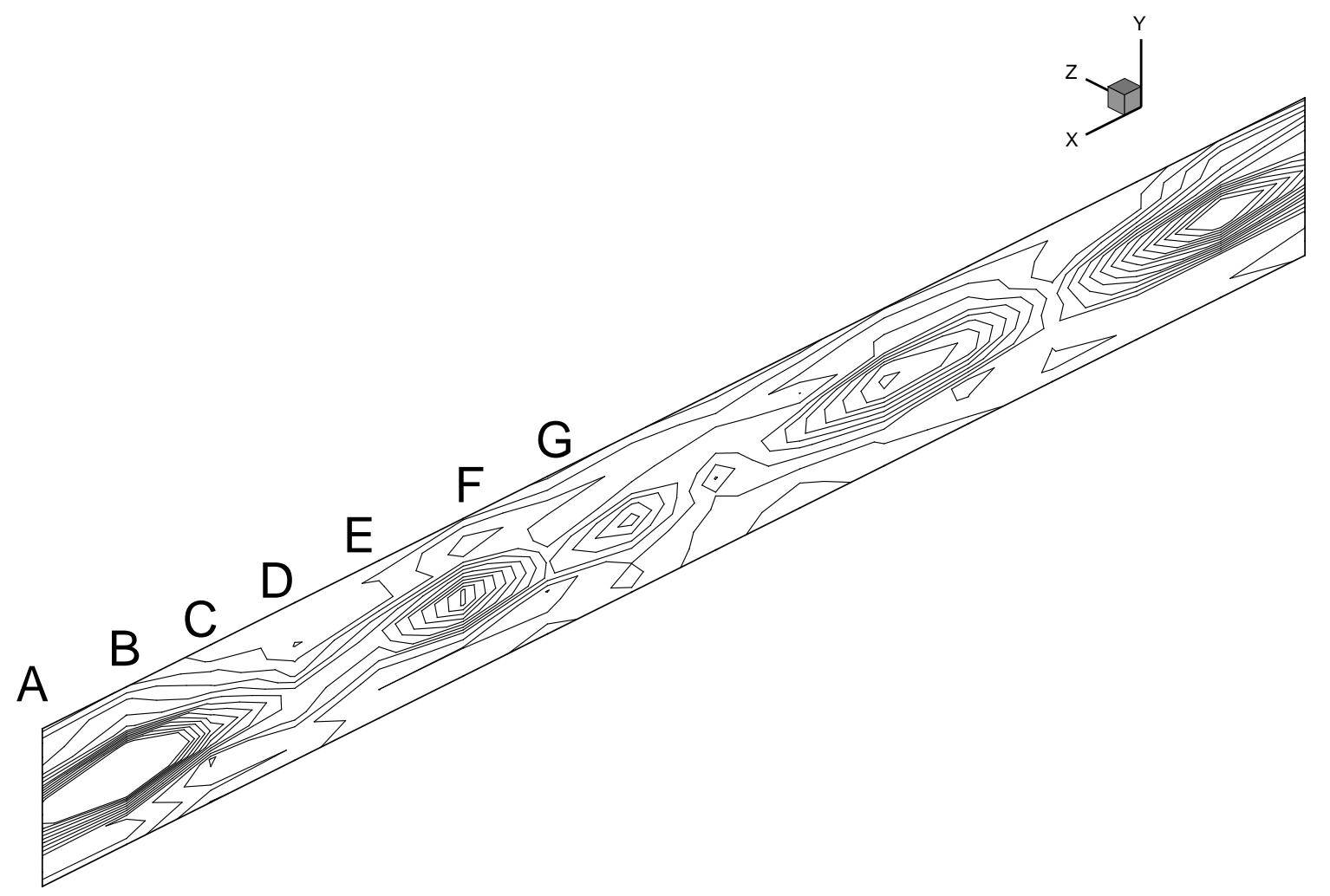

FigURE 26. Vorticity $\alpha=36.65$ for a filter setting of $(40,2)$ (38 mode system) $x_{1}, x_{2}$ plane. portance of including the feedback between the mean and turbulent flow. Then, two sets of equations have been presented, each with a different means of representing the mean streamwise velocity. In the first set of equations, a Boussinesq approximation was used to calculate the contribution from the truncation to the mean streamwise velocity. The mean velocity profile was then held constant for all time causing a fixed level of production. Several truncations were evaluated for this model. The first truncation involved 7 modes evenly spaced in $k_{1}$ for $k_{3}=0$. With this truncation, the system exhibited a rich range of 


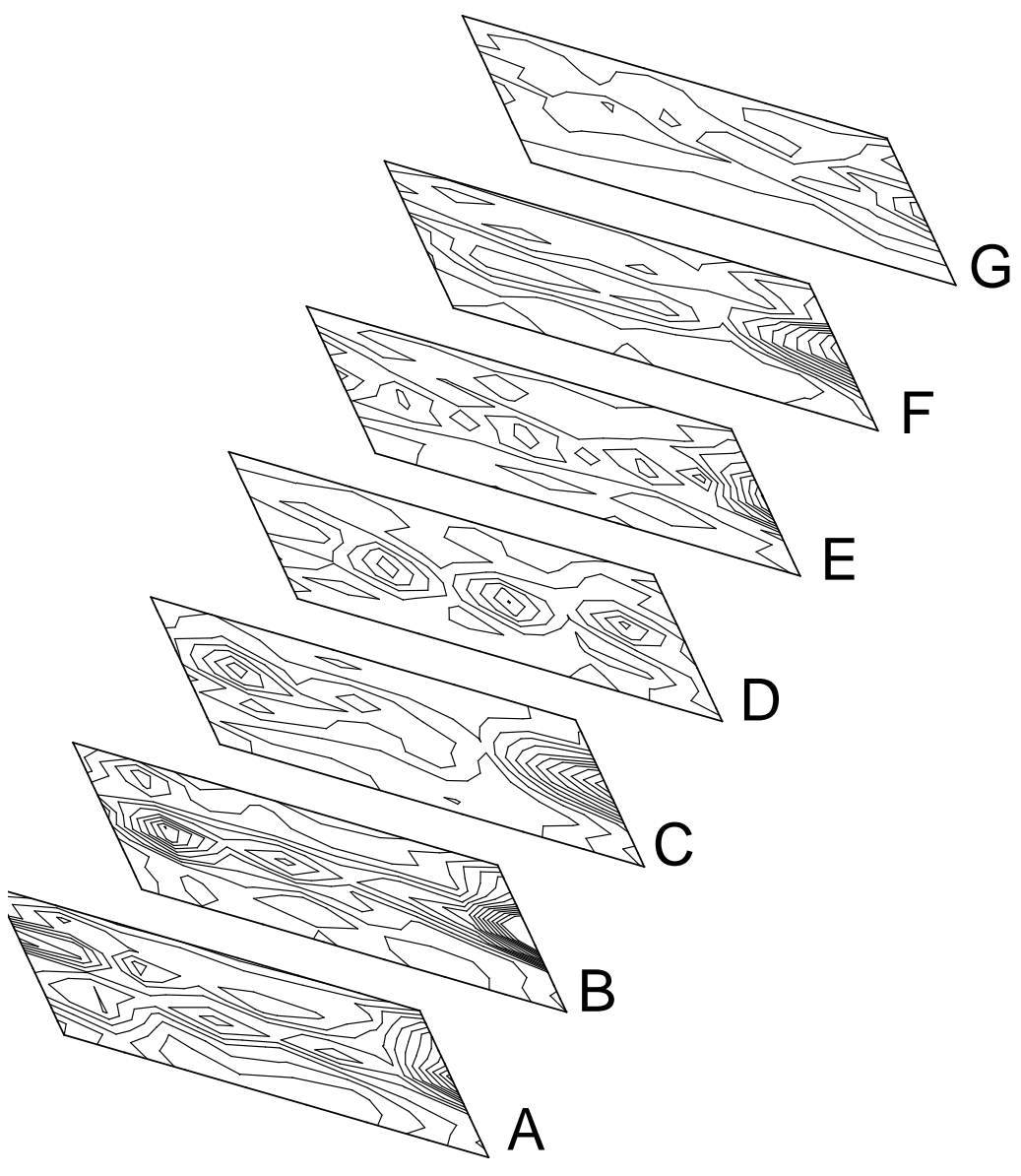

FiguRE 27. Vorticity $\alpha=36.65$ for a filter setting of $(40,2)$ (38 mode system) $x_{2}, x_{3}$ plane. dynamics. Along with this rich behaviour, an underlying periodicity was always present. This underlying periodicity, was at realistic frequencies for the truncation studied and is representative of the spanwise vortex tubes which are known to be a periodic event. While the complex behaviour on top of the underlying periodicity appears to control the structure interaction. The wavenumbers associated with the energy at the centre of the mixing layer tended to lead the temporal dynamics, which is consistent with the results of the stability analysis presented in $\S 6$ and those of Metcalfe et al. (1987). Different statistical quantities were checked for several values of the bifurcation parameter. The 
mean square velocity profiles displayed the correct $x_{2}$ distribution along with the proper magnitude ratio for the various component. The distribution of the spectra, from the simulations, also showed the correct trends in the $x_{2}$ direction. For large values of the bifurcation parameter, the velocity vector plots and spanwise vorticity contours showed the periodic spanwise structure passing through the window.

A truncation which involved spanwise wavenumbers not equal to zero was also examined. The time histories of the coefficients also exhibited a rich dynamical behaviour. The behaviour ranged from complex dynamics in full space to solutions which tended to revert back to a two dimensional solution. In the systems that exhibited complex dynamics in full space there was too much production which suggested that a scaling relationship (or filter) for the mean streamwise velocity would be useful.

The other set of equations that was studied allowed for both linear growth and feedback between the turbulence and the mean. In this method, a cutoff wavenumber was chosen and wavenumbers greater than this value are allowed to vary with time while the contribution from wavenumbers lesser than this value contribute in a non time dependent manner as with the first set of equations. Initial application of this method was encouraging. It served two purposes; firstly it allows feedback from between the turbulence, secondly it scales the mean velocity gradient which will in turn scale the contribution from the production term.

Solution of this equation for the 7 mode model had a definite effect the results. For filter settings which did not alter the mean streamwise velocity gradient the introduction of the cubic term served to stabilize the solutions. The system did not go unstable and exhibit a blow up condition regardless of the value of $\alpha$. 
Applying the Filter technique to the truncation involving non-zero spanwise wavenumbers also had a pronounced effect. The scaling of the mean streamwise velocity gradient reduced the production term thus resulting in magnitudes for the coefficients that were in good agreement with the experimentally determined ones. With the reduced energy in the system the amplitudes of the reconstructed mean square velocity profiles were consistent with what one would expect from the severely truncated system. Plots of the vorticity showed strong evidence of the known flow organizations. These plots showed spanwise vortex tubes being connected by streamwise aligned vorticity. This type of structure is consistent with what has been reported in many previous studies (see Metcalfe et al. 1987 for example).

This work was completed with the support of the French Ministry of Defense under the Grants DRET/DGA 90-171 and DRET/DGA 93.2549.A and a NSF/CNRS travel grant through the international program. Partial support for L. U. was provided by the French Ministry of Foreign Office under the Chateaubriand program. 


\section{Appendix A. General dynamical equations}

\section{A.1. No feedback relationship}

$$
\begin{aligned}
& \Lambda_{k_{1}, k_{3}}^{1(m)}=\nu\left[-4 \pi^{2}\left(k_{1}^{2}+k_{3}^{2}\right) \delta_{m n}+\int_{-L_{2}}^{L_{2}}\left(\frac{\mathrm{d}^{2} \Phi_{i, k_{1}, k_{3}}^{(m)}\left(x_{2}\right)}{\mathrm{d} x_{2}^{2}}\right) \Phi_{i, k_{1}, k_{3}}^{(n) *}\left(x_{2}\right) \mathrm{d} x_{2}\right] . \\
& \Lambda_{k_{1}, k_{3}}^{2(m)}=-\left(\int_{-L_{2}}^{L_{2}} \Phi_{2, k_{1}, k_{3}}^{(m)}\left(x_{2}^{\prime}\right) \frac{\partial U_{1}\left(x_{2}^{\prime}\right)}{\partial x_{2}} \Phi_{1, k_{1}, k_{3}}^{(n) *}\left(x_{2}^{\prime}\right) \mathrm{d} x_{2}^{\prime}+\left(2 \pi j k_{1}\right) \int_{-L_{2}}^{L_{2}} U_{1}\left(x_{2}^{\prime}\right) \Phi_{i, k_{1}, k_{3}}^{(m)}\left(x_{2}^{\prime}\right) \Phi_{i, k_{1}, k_{3}}^{(n) *}\left(x_{2}^{\prime}\right) \mathrm{d} x_{2}^{\prime}\right) \\
& Q_{k_{1}^{\prime}, k_{3}^{\prime}, k_{1}, k_{3}}^{(p)(q)}=-\frac{\left(1-\delta_{k_{1}, 0} \delta_{k_{3}, 0}\right)}{\left(L_{1} L_{3}\right)^{\frac{1}{2}}} \times \int_{-L_{2}}^{L_{2}}[ \\
& 2 \pi \jmath\left(k_{1}-k_{1}^{\prime}\right) \Phi_{1, k^{\prime}{ }_{1}, k^{\prime}{ }_{3}}^{(p)}\left(x_{2}\right) \Phi_{i, k_{1}-k^{\prime}{ }_{1}, k_{3}-k_{3} \prime_{3}}^{(q)}\left(x_{2}\right)+ \\
& \Phi_{2, k^{\prime}{ }_{1}, k^{\prime} 3}^{(p)}\left(x_{2}\right) \frac{\mathrm{d}}{\mathrm{d} x_{2}} \Phi_{i, k_{1}-k^{\prime}{ }_{1}, k_{3}-k_{3}^{\prime}{ }_{3}}^{(q)}\left(x_{2}\right)+ \\
& 2 \pi \jmath\left(k_{3}-k^{\prime}{ }_{3}\right) \Phi_{3, k^{\prime}{ }_{1}, k^{\prime}{ }_{3}}^{(p)}\left(x_{2}\right) \Phi_{i, k_{1}-k^{\prime}{ }_{1}, k_{3}-k_{3}{ }_{3}}^{(q)}\left(x_{2}\right) \\
& ] \Phi_{i, k_{1}, k_{3}}^{(n) *}\left(x_{2}\right) \mathrm{d} x_{2}
\end{aligned}
$$

\section{A.2. Filter relationship}

$$
\begin{aligned}
& C_{k_{1}^{\prime}, k_{3}^{\prime}, k_{1}, k_{3}}^{(p)(q)(r)}=\frac{1}{\nu_{e}\left(L_{1} L_{3}\right)}\left[\int _ { - L _ { 2 } } ^ { L _ { 2 } } \left[\left(\int_{0}^{x_{2}} \Phi_{1, k_{1}^{\prime}, k_{3}^{\prime}}^{(p)}\left(x_{2}^{\prime}\right) \Phi_{2, k_{1}^{\prime}, k_{3}^{\prime}}^{(q) *}\left(x_{2}^{\prime}\right) \mathrm{d} x_{2}^{\prime}\right)\left(2 \pi j k_{1}\right) \Phi_{i, k_{1}, k_{3}}^{(r)}\left(x_{2}\right)\right.\right. \\
& \left.\left.+\delta_{i 1}\left(\Phi_{1, k_{1}^{\prime}, k_{3}^{\prime}}^{(p)}\left(x_{2}\right) \Phi_{2, k_{1}^{\prime}, k_{3}^{\prime}}^{(q) *}\left(x_{2}\right) \Phi_{2, k_{1}, k_{3}}^{(r)}\left(x_{2}\right)\right)\right] \Phi_{i, k_{1}, k_{3}}^{(n) *}\left(x_{2}\right) \mathrm{d} x_{2}\right] .
\end{aligned}
$$




\section{Appendix B. Turbulent viscosity model}

In Aubry et al. (1988) this assumption is written in the following form:

$$
\tau_{i j}^{S S}=-2 \nu_{T} S_{i j}^{L, S}
$$

denoting that the small scale stress tensor is proportional to the strain rate tensor for the modes resolved. In this equation, $\tau_{i j}^{S S}$ and $S_{i j}^{L S}$ are defined as:

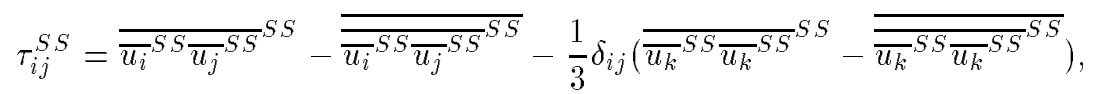

and

$$
S_{i j}^{L S}=\frac{1}{2}\left(\frac{\partial \bar{u}_{i}^{L S}}{\partial x_{j}}+\frac{\partial{\overline{u_{j}}}^{L S}}{\partial x_{i}}\right),
$$

where the notation $\overline{()}^{L S}$ (Large Scale) corresponds to the summation over all the modes and wavenumbers inferior or equal to the truncation point; the notation $\overline{(~)}^{\text {SS }}$ (Small Scale) corresponds to the small scales (summation over all the modes wavenumbers larger than the truncation point). By observation that the energy decreases rapidly with higher POD and streamwise/spanwise wavenumber modes (see the $3 \mathrm{D}$ eigenspectra represented in Delville et al. 1998), the assumption can be made that the relevant scales are given by characteristic scales of the first neglected modes.

With these conditions the turbulent viscosity can be defined as follows:

$$
\nu_{T}=\frac{\int_{\mathcal{D}} \overline{u_{i}^{S S} u_{i}^{S S}} \mathrm{~d} x_{2}}{\left(\mathcal{D} \int_{\mathcal{D}} \overline{\frac{\partial u_{i}^{S S}}{\partial x_{j}} \frac{\partial u_{i}^{S S}}{\partial x_{j}}} \mathrm{~d} x_{2}\right)^{\frac{1}{2}}} .
$$

The integrals over $x_{2}$ are used to eliminate the inhomogeneous direction since $\nu_{T}$ should not be a function of this direction. In (B 4), the term $-\frac{1}{3} \delta_{i j}\left(\overline{\overline{u_{k}} S S \overline{u_{k}} S S} S S-\overline{\overline{u_{k}} S S \overline{u_{k}} S S}\right.$ ) has been dropped because it can be combined with the pressure term which has been dropped in this analysis (see $\S 3.3$ ). This representation is simpler than that of Aubry et 
al. (1988) since it was necessary in that study to keep the pressure term, but is the same of that of Glauser et al. (1989).

Utilizing the relationships of (2.4) and (3.11), the following expression arises:

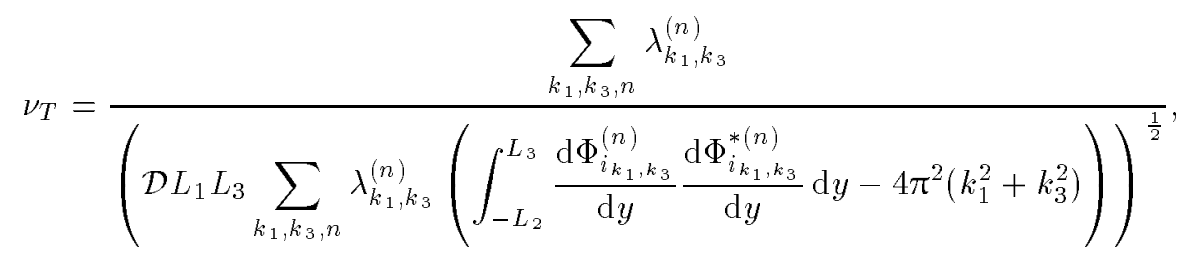

where the sum $\sum_{k_{1}, k_{3}, n}$ corresponds only to the first neglected modes. 


\section{Appendix C. Wavenumber pairs and initial conditions}

C.1. 7 mode model

\begin{tabular}{ll}
$\left(k_{1}, k_{3}\right)$ & $a_{k_{1}, k_{3}}(t=0)$ \\
$(10,0)$ & $7.6000010^{-03}$ \\
$(20,0)$ & $1.1377110^{-02}$ \\
$(30,0)$ & $1.7275510^{-02}$ \\
$(40,0)$ & $2.0643110^{-02}$ \\
$(50,0)$ & $1.7252410^{-02}$ \\
$(60,0)$ & $8.7966410^{-03}$ \\
$(70,0)$ & $5.0021010^{-03}$ \\
\hline
\end{tabular}

Table 4: Wavenumber pairs and initial conditions for 7 mode model.

C.2. 38 mode model

\begin{tabular}{|c|c|c|c|c|c|c|}
\hline$\left(k_{1}, k_{3}\right)$ & $(10,0)$ & $(20,0)$ & $(30,0)$ & $(40,0)$ & $(50,0)$ & \\
\hline$a_{k_{1}, k_{3}}(t=0)$ & $7.6000010^{-03}$ & $1.1377110^{-02}$ & $1.7275510^{-02}$ & $2.0643110^{-02}$ & $1.7252410^{-02}$ & \\
\hline$\left(k_{1}, k_{3}\right)$ & $(0,1)$ & $(10,1)$ & $(20,1)$ & $(30,1)$ & $(40,1)$ & $(50,1)$ \\
\hline$a_{k_{1}, k_{3}}(t=0)$ & $6.8004010^{-03}$ & $7.5392610^{-03}$ & $1.2097110^{-02}$ & $1.9660810^{-02}$ & $2.4208510^{-02}$ & $2.0748410^{-02}$ \\
\hline$\left(k_{1}, k_{3}\right)$ & $(0,2)$ & $(10,2)$ & $(20,2)$ & $(30,2)$ & $(40,2)$ & $(50,2)$ \\
\hline$a_{k_{1}, k_{3}}(t=0)$ & $7.1037110^{-03}$ & $7.0966010^{-03}$ & $9.4114210^{-03}$ & $1.2601310^{-02}$ & $1.4384910^{-02}$ & $1.1807910^{-02}$ \\
\hline$\left(k_{1}, k_{3}\right)$ & $(0,3)$ & $(10,3)$ & $(20,3)$ & $(30,3)$ & $(40,3)$ & $(50,3)$ \\
\hline$a_{k_{1}, k_{3}}(t=0)$ & $6.7477310^{-03}$ & $6.5809910^{-03}$ & $8.2058910^{-03}$ & $9.5702910^{-03}$ & $1.0279010^{-02}$ & $8.2903410^{-03}$ \\
\hline
\end{tabular}

Table 5: Wavenumber pairs and Initial conditions for 38 mode model. 


\section{REFERENCES}

Aubry, N., Holmes, P., Lumley, J.L. \& Stone, E. 1988 The dynamics of coherent structures in the wall region of a turbulent boundary layer. J. Fluid Mech. 192, 115-173.

Aubry, N., Lumley, J.L. \& Holmes, P. 1990 The Effect of modeled drag reduction on the wall region. Theoret. Comput. Fluid Dynamics 1, 229-248.

Berkooz, G. 1992 Feedback control of boundary deformation of models of the turbulent wall layer. Report FDA-92-16, Cornell University.

Berkooz, G., Holmes, P. \& Lumley, J.L. 1991 Intermittent dynamics in simple models of the turbulent wall layer. J. Fluid Mech. 230, 75-95.

Berkooz, G., Holmes, P. \& Lumley, J.L. 1993a The Proper Orthogonal Decomposition in the analysis of turbulent flows. Ann. Rev. Fluid Mech. 25, 539-575.

Berkooz, G., Holmes, P. \& Lumley, J.L. 1993b On the relation between low-dimensional models and the dynamics of coherent structures in turbulent wall layers. Theor. Comput. Fluid Dynamics 4, 255-269.

Boussinesq, T.V. 1877 Théorie de l'écoulement tourbillonnant. Mém. Pré. par div. Sav. Paris, 23.

Citriniti, J.H. 1996 Experimental investigation into the dynamics of the axisymmetric mixing layer utilizing the Proper Orthogonal Decomposition. PhD thesis, State University of New York at Buffalo.

CoRDIER, L. 1996 Etude de systèmes dynamiques baseés sur la décomposition orthogonale aux valeurs propres (POD). Application à la couche de mélange turbulente et à l'écoulement entre deux disques contra-rotatifs. PhD thesis, University of Poitiers.

Delville, J. 1995 La décomposition orthogonale aux valeurs propres et l'analyse de l'organisation tridimensionelle des écoulements turbulents cisaillés libres. PhD thesis, University of Poitiers.

Delville, J., Ukeiley, L.S., Bonnet, J.-P. \& Glauser, M.N. 1998 Examination of LargeScale Structures in a Turbulent Plane Mixing Layer. Part 1. Proper Orthogonal Decomposition. Under consideration for publication in J. Fluid Mech.. 
Doedel, E.J., Keller, H. \& Kernevez J.P. 1991 Numerical analysis and control of bifurcation problems. International Journal of Bifurcation and Chaos, 1, $N^{\circ} 3,493-520$.

George, W.K. 1988 Insight into the dynamics of coherent structures from a Proper Orthogonal Decomposition. In Symposium from near wall turbulence, Dubrovnik, Yugoslavia, May 1620.

GLauser, M.N. 1987 Coherent structures in the axisymmetric turbulent jet. PhD thesis, State University of New York at Buffalo.

Glauser, M.N., Zheng, X. \& Doering C.R. 1989 The dynamics of organized structures in the axisymmetric jet mixing layer. In Turbulence and Coherent Structures, (ed. O. Metais \& M. Lesieur). Kluwer Academic Publishers.

Glauser, M.N., Zheng, X. \& George, W.K. 1990 The streamwise evolution of coherent structures in the axisymmetric jet mixing layer. In Recent developments in turbulence, (ed. Gatski, Sarkar \& Speziale). Springer.

Glauser, M.N. \& George, W.K. 1992 Application of multipoint measurements for flow characterization. Experimental Thermal and Fluid Sciences 5, 617-632.

Herzog, S. 1986 The large-scale structure in the near-wall of turbulent pipe flow. PhD thesis, Cornell University.

HinZE, J.O. 1975 Turbulence. McGraw-Hill, Inc.

Holmes, P. 1990 Can dynamical systems approach turbulence ?. In Whither Turbulence? Turbulence at the Crossroads. (ed. J.L. Lumley). Springer.

Holmes, P, Lumley, J.L. \& Berkooz, G. 1996 Turbulence, Coherent Structures, Dynamical Systems and Symmetry. Cambridge University Press.

Lorenz, E.N. 1963 Deterministic non-periodic flow. J. Atmos. Sciences 20, 130-141.

Lumley, J.L. 1967 The structure of inhomogeneous turbulent flows. Atm. Turb. and Radio Wave Prop.. (ed. Yaglom and Tatarsky). Nauka, Moscow, 166-178.

Lumley, J.L. 1970 Stochastic Tools in Turbulence. Academic Press.

Metcalfe, R.W., Orszag, S.A., Brachet, M.E., Menon, M. \& Riley, J.J. 1987 Secondary instability of a temporally growing mixing layer. J. Fluid Mech., 184, 207-243. 
Michalke, A. 1964 On the inviscid instability of the hyperbolic-tangent velocity profile. $J$. Fluid Mech. 19 part 4, 543-556.

Pierrehumbert, R.T. \& Widnall, S.E. 1982 The two- and three-dimensional instabilities of a spatially periodic shear layer. J. Fluid Mech. 114, 59-82.

Rajaee, M., Karlsson, S. \& Sirovich, L. 1994 Low-dimensional description of free-shear-flow coherent structures and their dynamical behaviour. J. Fluid Mech., 258, 1-29.

REMPFER, D. 1995 Investigations of boundary layer transition via Galerkin projections of empirical eigenfunctions. Phys. Fluids 8 (1), 175-188.

Ruelle, D. \& Takens, F. 1971 On the nature of turbulence. Communs. Math. Phys. 20, $167-192$.

SANGHi, S. \& Aubry, N. 1993 Mode interaction models for near-wall turbulence. J. Fluid Mech., 252, 239-264.

UKEILEY, L.S. 1995 Dynamics of large-scale structures in a plane turbulent mixing layer. PhD thesis, Clarkson University.

Wick, D.P., Glauser, M.N. \& Ukemey, L.S. 1994 Investigation of turbulent flows via Pseudo Flow Visualization. Part 1. Axisymmetric jet mixing layer. Experimental Thermal and Fluid Sciences 9, 391-404

Zheng, X. \& Glauser M.N. 1991 A low-dimensional dynamical systems description of coherent structures in the axisymmetric jet mixing layer. Report No. MAE-247, Clarkson University.

Zhou, X. \& SiRovich, L. 1992 Coherence and chaos in a model of turbulent boundary layer, Phys. Fluids A 4 (12), 2855-2874. 
6.1. Analysis method 
A.1. No feedback relationship 